\title{
Prognostic and Predictive Value of Blood Tumor Mutational Burden in Patients With Lung Cancer Treated With Docetaxel
}

\author{
Wei Nie, MD1,*; Jie Qian, MD1,*; Mi-Die Xu, MD2,*; Kai Gu, MD,*; Fang-Fei Qian, MD; Jun Lu, MD;

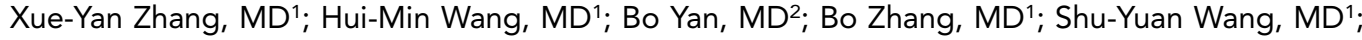 \\ Fang $\mathrm{Hu}, \mathrm{MD}^{1}$; Chang-Hui Li, MD${ }^{1}$; Hua Zhong, $\mathrm{MD}^{1}$; and Bao-Hui Han, MD ${ }^{1}$
}

\begin{abstract}
Background: Biomarkers for chemotherapy efficacy in non-small cell lung cancer (NSCLC) are lacking. This retrospective study assesses the association between blood-based tumor mutational burden (bTMB) and clinical benefit of chemotherapy. Methods: Clinical and targeted next-generation sequencing data from the OAK trial (training set; $n=318$ ) and POPLAR trial (validation set; $n=106$ ) in the docetaxel arm were analyzed. The cutoff value of bTMB for outcome prediction was determined based on a time-dependent receiver operating characteristic curve in the training set, and propensity score matching (PSM) was conducted. The primary outcome was overall survival (OS). Durable clinical benefit (DCB) was defined as OS lasting $>12$ months. Interaction between treatment and bTMB was assessed in the combined set. Results: A lower bTMB was observed in patients with DCB compared with no durable benefit, and in those with a partial response and stable disease compared with progressive disease. The optimized cutoff value of bTMB for predicting OS was 7 single-nucleotide variants per megabase. In the training set, a low bTMB was significantly associated with longer OS and progressionfree survival (PFS). The prognostic value of bTMB was confirmed in the validation set and PSM set. The interaction between $b T M B$ and treatment was significant for PFS (interaction $P=.043$ ) in the combined set. Mutations in KEAP1 were associated with high $b T M B$ and a lack of benefit from chemotherapy. Conclusions: Low bTMB is associated with a survival advantage in patients with NSCLC treated with docetaxel, suggesting the prognostic and predictive potential of bTMB for determining chemotherapy efficacy.
\end{abstract}

J Natl Compr Canc Netw 2020;18(5):582-589 doi: $10.6004 /$ jnccn.2019.7383

${ }^{1}$ Shanghai Chest Hospital, Shanghai Jiao Tong University; ${ }^{2}$ Fudan University Shanghai Cancer Center; and ${ }^{3}$ Shanghai Applied Protein Technology Co, Ltd (APTBIO), Shanghai, China.

\section{Background}

For treatment of advanced non-small cell lung cancer (NSCLC) without driver mutations or high PD-Ll expression, chemotherapy remains the standard of care. However, the clinical benefit of chemotherapy is limited, with the objective response rate (ORR) between $20 \%$ and $30 \%$ for first-line treatment and approximately $10 \%$ for second-line treatment. ${ }^{1-3}$ Thus, identification of a subset of patients who could gain clinical benefit from chemotherapy is a critical unmet need.

In the past 2 decades, many candidate biomarkers have been investigated. However, no validated biomarker for chemotherapy customization could be used in the clinical setting. Lee et $\mathrm{al}^{4}$ showed that both excision repair cross-complementing group 1 (ERCC1) and XPF protein expression could not predict clinical outcomes for platinum therapy in advanced NSCLC. Bepler et $\mathrm{al}^{5}$ failed to find a significant correlation between disease response and protein or mRNA ERCC1 or ribonucleotide reductase messenger 1 (RRM1) levels in patients with advanced NSCLC treated with gemcitabine/carboplatin. In addition, Seymour et $\mathrm{al}^{6}$ concluded that most of the promising biomarkers could not be validated in the Lung Adjuvant Cisplatin Evaluation (LACE)-Bio project.

Tumor mutational burden (TMB) is emerging as a new biomarker for predicting response to immune checkpoint inhibitors. ${ }^{7}$ Blood-based tumor mutational burden (bTMB), which is measured with targeted nextgeneration sequencing (NGS) using cell-free DNA, shows a positive correlation with tissue-based TMB and is a biomarker for better progression-free survival (PFS) in patients with NSCLC receiving atezolizumab. ${ }^{8}$ However, the role of bTMB in other treatment settings, such as chemotherapy, is unknown. In chemotherapy, diverse genetic mutations may result in resistance to therapy. ${ }^{9}$ We thus hypothesized that low bTMB would be a

See JNCCN.org for supplemental online content. 
favorable prognostic biomarker for patients with advanced NSCLC treated with chemotherapy. To test this hypothesis, clinical and genetic data from the OAK and POPLAR trials were analyzed. We identified a bTMB cutpoint in the OAK trial, and validated its prognostic value in the POPLAR trial.

\section{Methods}

\section{Study Design and Patient Population}

The design of the randomized, open-label, international OAK and POPLAR trials has been described previously. ${ }^{8,10,11}$ Patients who had previously received chemotherapy for stage IIIB or IV NSCLC were randomized (1:1) to treatment with atezolizumab (1,200 mg fixed dose) or docetaxel $\left(75 \mathrm{mg} / \mathrm{m}^{2}\right)$. Because the aim of this study was to investigate how bTMB impacted survival of patients with advanced NSCLC treated with chemotherapy, only docetaxel arms from the OAK (training set; $n=318$ ) and POPLAR trials (validation set; $n=106$ ) were included. The atezolizumab arms from 2 trials (non-chemotherapy-treated NSCLC; $\mathrm{n}=324$ ) were included as a comparison group. ${ }^{10,11}$ No Institutional Review Board approval was required, because all of the clinical and bTMB data were obtained from a published study by Gandara et al. ${ }^{8}$

\section{Outcomes}

The primary outcome was overall survival (OS), which was defined as the time from treatment initiation until death resulting from any cause. PFS was assessed from chemotherapy initiation until disease progression or death from any cause. Durable clinical benefit (DCB) was defined as OS lasting $>12$ months, whereas no durable benefit (NDB) was defined as OS lasting $<12$ months. RECIST version 1.1 was used to assess efficacy.

\section{Next-Generation Sequencing}

All patients had NGS testing performed by FoundationOne as described previously. ${ }^{12,13}$ Blood samples were sequenced on a 394-gene panel, which included 1.1 Mb of coding region in the genome. ${ }^{8}$ To calculate bTMB score, the total number of mutations was divided by the coding region. The germline mutations were filtered out by comparing against the dbSNP and ExAC databases.

\section{Statistical Analysis}

Mann-Whitney $U$ test was used to examine the difference between 2 groups. Fisher exact test was used to evaluate between-group differences for proportions. Receiver operating characteristic (ROC) curve that plotted sensitivity against 1-specificity of bTMB and rate of DCB was assessed by generating the area under the curve (AUC) for the training set. The accuracy of bTMB for predicting OS was assessed using time-dependent ROC in the training set. ${ }^{14}$ The cutoff value of bTMB was determined by the highest Youden index (sensitivity + specificity - 1). Probability of survival over time was assessed using Kaplan-Meier estimation, and Cox proportional hazards regression was used to estimate hazard ratio (HR) and 95\% CI. Multivariate Cox proportional hazards model analysis was performed integrating age, race, sex, smoking status, ECOG performance status, histology, treatment line, baseline sum of longest diameters, number of metastatic sites, PD-L1 expression (training set only), driver mutations (training set only), and bTMB groups. Subgroup analyses were performed according to age, race, sex, smoking status, ECOG performance status, histology, treatment line, baseline sum of longest diameters, number of metastatic sites, PD-L1 expression (training set only), and driver mutations (training set only). To adjust for selection bias, a propensity score was assessed for all patients (training and validation sets) ${ }^{15}$ because of the imbalanced data between low and high bTMB groups. Logistic regression was used for estimating the propensity scores, with a caliper approach as 0.2 of the standard deviation. Nearest neighbor matching was selected, and matched controls were not replaced during matching. Using the propensity scores, patients with high bTMB were randomly matched to those with low bTMB using a 1:1 matching method. Because some covariates remained imbalanced after matching, we used doubly robust estimation to ensure comprehensive adjustment. The prevalence of dichotomous variables was compared between treated and untreated subjects using chi-square test, whereas a standard 2 -sample $t$-test was used to compare continuous baseline covariates. Treatment-bTMB interaction was tested in the combined set (training + validation) as an exploratory analysis.

An unbiased analysis of enrichment in frequency of altered genes within individual groups were examined by plotting the $\log 2$ (odds ratio) or HR versus $\log 2$ (Fisher exact test $P$ value or univariate Cox proportional hazards regression $P$ value for OS and PFS). False discovery rate (FDR) was used to correct for multiple comparisons $(\mathrm{q}<0.1)$. All tests were 2-sided at a significance level of 0.05 . All statistical analyses were performed using $R$ version 3.3.1 (R Foundation for Statistical Computing) and STATA, version 12.0 (StataCorp LLP).

\section{Results}

\section{Clinical Characteristics of the Patient Population}

The process of patient selection is shown in supplemental eFigure 1 (available with this article at JNCCN. org). A total of 425 and 143 patients treated with docetaxel were identified from the OAK and POPLAR trials, 
respectively, with 107 and 37 patients removed based on certain criteria. Therefore, the final training and validation sets included 318 and 106 patients, respectively. No significant differences in patient characteristics were seen between the training and validation sets, except for race (supplemental eTable 1). Median follow-up was 21 months for the training set and 15.7 months for the validation set.

\section{Prognostic Value of bTMB in the Training Set}

In the training set, the bTMB score was lower in patients with DCB than in those with NDB (median, 5 vs 9 singlenucleotide variants [SNVs] /megabase [Mb]; $P<.001$ ), and it was associated with response to docetaxel (partial response vs stable disease vs progressive disease, median bTMB: 5 vs 7 vs 10 SNVs/Mb, respectively; $P=.007$; Figure 1A). We then investigated the relationship between the cutpoints of bTMB and rates of DCB. When TMB was stratified into increasing quartiles, rates of $\mathrm{DCB}$ decreased with increasing bTMB (supplemental eFigure 2). A significantly higher DCB rate was found in those with bTMB below versus above the 50th percentile (bTMB $=7 \mathrm{SNVs} / \mathrm{Mb}$; DCB rate, $44.7 \%$ vs $15.9 \%$, OR, 2.72 , $P<.001$ ) (Figure $1 \mathrm{~B}$ and supplemental eFigure 2). The bTMB-based DCB classifier had an AUC of 0.628 in the ROC curve, which indicated an optimal bTMB cutoff value of 7.5 $\mathrm{SNVs} / \mathrm{Mb}$ (approximately equal to $7 \mathrm{SNVs} / \mathrm{Mb}$ ) to predict DCB, with a sensitivity of $57.5 \%$ and specificity of $67.3 \%$ (Figure 1C). The optimized cutoff value of bTMB for predicting 2-year OS was $7 \mathrm{SNVs} / \mathrm{Mb}$ by time-dependent ROC curve (Figure 1D). Therefore, the bTMB of 7 SNVs/Mb was used as the cutoff point in the following analyses.

Clinical characteristics for the low $(\leq 7 \mathrm{SNVs} / \mathrm{Mb})$ and high bTMB ( $>7 \mathrm{SNVs} / \mathrm{Mb}$ ) groups of the training set are shown in supplemental eTable 2. Median OS of patients with low bTMB was 11.5 versus 6.4 months for those with high bTMB (HR, 0.638; 95\% CI, 0.497-0.820; $P<.001$; Figure 2A). Median PFS in the low bTMB group (4.3 months) was significantly longer compared with the high bTMB group (2.9 months; HR, 0.588; 95\% CI, $0.466-0.742 ; P<.001$; Figure 2B). These differential effects were confirmed in the multivariate analyses (supplemental eTable 3). Across most subgroups, results of the analyses of OS and PFS were consistent with the overall results (Figure 2C, D). Although the ORR for the low bTMB group was only numerically higher compared with the high bTMB group (17.1\% vs $11.7 \% ; P=.209$ ), the disease control rate (DCR) was significantly higher among patients with low bTMB (71.9\% vs $56.2 \% ; P=.007)$ (supplemental eFigure 3A).

\section{Prognostic Value of bTMB in the Validation Set}

The prognostic value of the cutoff point (bTMB $=7$ $\mathrm{SNVs} / \mathrm{Mb}$ ) was assessed in the validation set (supplemental eTable 4). Multivariate analysis showed shorter
OS and PFS among patients with high bTMB (Figure 3 and supplemental eTable 5). Subgroup analyses showed no noticeable difference (Figure 3C, D). In addition, in the validation and combined sets, significantly higher ORRs and DCRs were observed in low bTMB groups (supplemental eFigure 3B, C).

\section{Prognostic Value of bTMB in the Propensity Score-Matched Set}

Details of each confounding variable before and after matching are summarized in supplemental eTable 6 . After matching, baseline characteristics were generally comparable between patients with low $(n=129)$ and high bTMB $(n=129)$, except for smoking status, baseline sum of longest diameters, and EGFR mutation. High bTMB was significantly associated with shorter OS and PFS (supplemental eFigure 4A, B), with doubly robust estimation confirming these results (supplemental eTable 7). Because EGFR tyrosine kinase inhibitors (TKIs) could increase the OS rate among patients with low TMB, we analyzed again after excluding patients with EGFR mutations. We also found that higher bTMB was significantly correlated with shorter OS and PFS (data not shown).

Collectively, these results revealed that patients with NSCLC and low bTMB had longer PFS and OS than those with high bTMB.

\section{Treatment-bTMB Interaction in the Combined Set} The treatment-bTMB interaction was not significant for OS in the combined set (interaction $P=.777$; supplemental eTable 8). However, the interaction between bTMB and treatment was significant for PFS (interaction $P=.043$ ), suggesting the predictive role of bTMB for chemotherapy benefit.

\section{Individual Gene Alterations and Response to Chemotherapy}

We next evaluated whether mutations in individual genes were associated with resistance to chemotherapy and duration of benefit in the combined set $(n=424)$. Genetic variants in cell-free DNA differed between low and high bTMB groups (supplemental eFigure 5) and among partial response, progressive disease, and stable disease groups (supplemental eFigure 6). The mutations of 11 genes, such as KEAP1, were mostly enriched in the high bTMB group (supplemental eFigure 7) and associated with significantly shorter PFS or OS (FDR-adjusted $P<.100$; supplemental eFigure $6 \mathrm{C}, \mathrm{D}$ ).

KEAP1 mutations were more common among patients with NDB versus DCB (46/269, $17.1 \%$ vs $10 / 138,7.2 \%$; $P=.019$ ), and concurrent $E G F R$ mutations were more common in those with DCB $(20 / 138,14.5 \%$ vs $13 / 269$, $4.8 \%$ for NDB; $P=.001$ ) (supplemental eFigure $8 \mathrm{~A}, \mathrm{~B}$ ). 
A

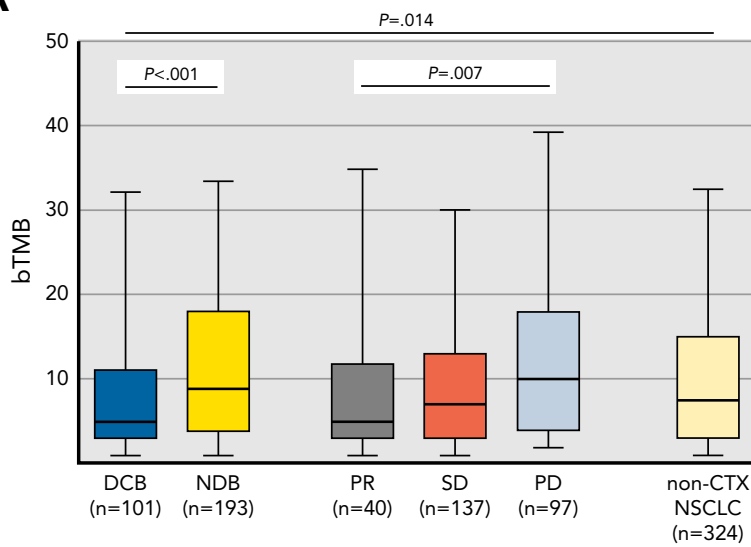

B

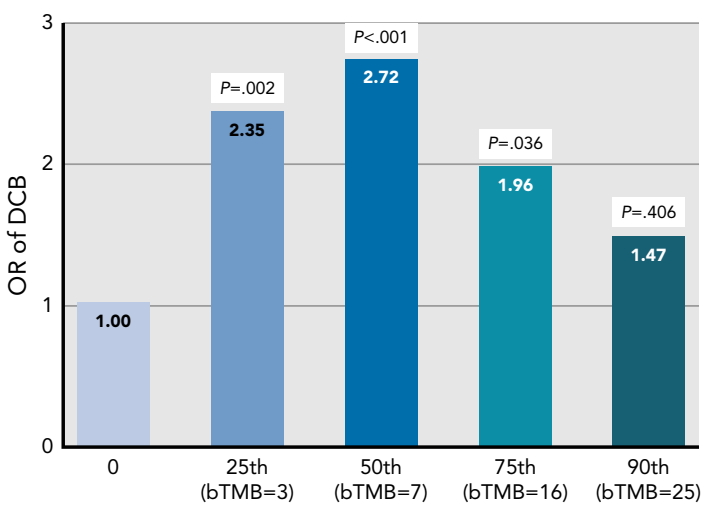

C

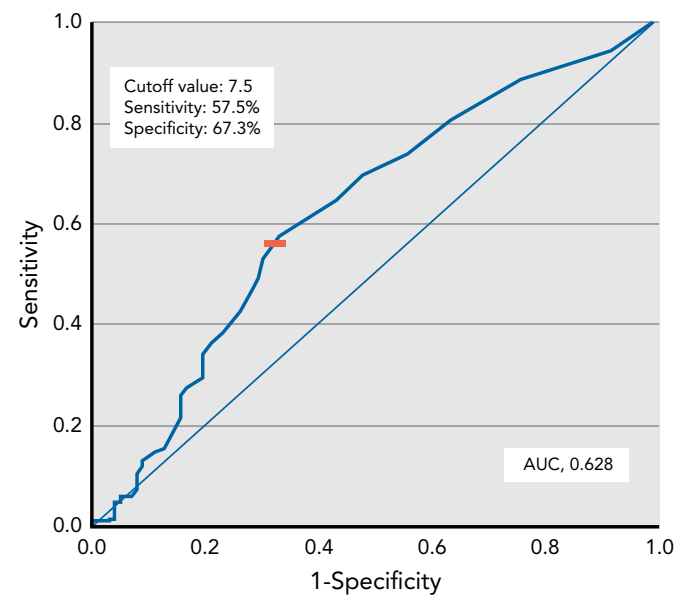

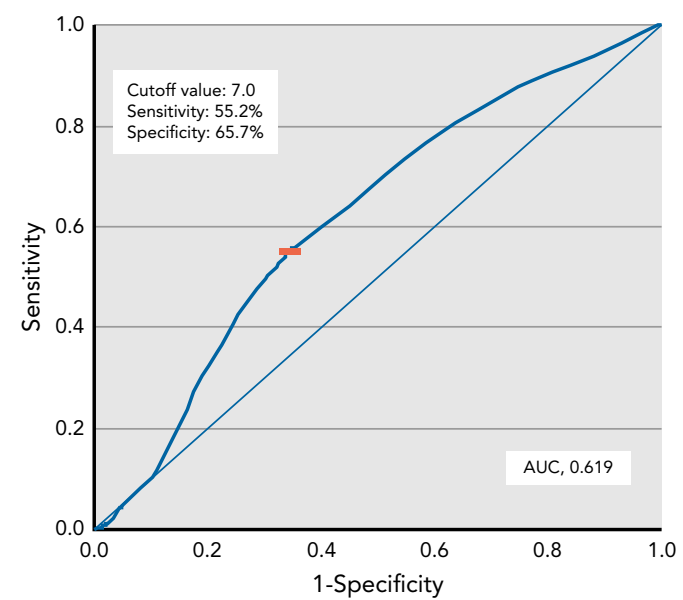

Figure 1. Somatic molecular features associated with response to chemotherapy. (A) bTMB is greater in NDB versus DCB (median, 9 vs 5 SNVs/Mb; $P<.001$ ) and is significantly different in those with $P R$ versus SD versus $P D$ (median, 5 vs 7 vs $10 \mathrm{SNVs} / \mathrm{Mb} ; P=.007$ ). The distribution of bTMB in patients with non-CTX-treated NSCLC are shown for reference. TMB in patients with DCB was similar to those with PR and greater in those with non-CTX-treated NSCLC ( $P=.014)$. Box plots represent medians and interquartile ranges, and vertical lines extend to the 95th percentiles. (B) OR of DCB with increasing cutpoints of TMB. The 0 percentile is shown for reference of all patients (default OR, 1.00). Odds of DCB increase significantly at the 50th percentile of TMB. (C) ROC curve of sensitivity versus 1-specificity of DCB at varying levels of bTMB (cutoff value: 7.5 ; AUC, 0.628). (D) Time-dependence ROC curve of sensitivity versus 1-specificity of 2-year OS at varying levels of bTMB (cutoff value: 7.0; AUC, $0.619)$.

Abbreviations: AUC, area under the curve; bTMB, blood-based tumor mutational burden; CTX, chemotherapy; DCB, durable clinical benefit; Mb, megabase; NCSLC, non-small cell lung cancer; NDB, no durable benefit; OR, odds ratio; PD, progressive disease; PR, partial response; ROC, receiver operating characteristic; SD, stable disease; SNV, single-nucleotide variant; TMB, tumor mutational burden.

KEAP1 and EGFR mutations were enriched in NDB and DCB groups, but these associations were not statistically significant (FDR-adjusted $P>.1$ ) (supplemental eFigure $8 \mathrm{C})$.

The location of KEAP1 mutations is shown in supplemental eFigure 9A. Patients with KEAP1 mutations had significantly higher bTMB (median, 16 vs 7 SNVs/ $\mathrm{Mb} ; P<.001$; supplemental eFigure 9B). KEAP1 mutation was significantly correlated with shorter PFS (2.7 vs 4.0 months; HR, 1.544; 95\% CI, 1.164-2.048; $P=.002$ ) and OS (5.8 vs 9.4 months; HR, 1.910; 95\% CI, 1.412-2.584; $P<.001$ ) (supplemental eFigure 9C, D). Consequently, patients with NSCLC and KEAP1 mutations could not derive benefit from chemotherapy.

\section{Median Allele Frequency and Survival Outcomes}

We further evaluated the association between median allele frequency (MAF) of variants and bTMB and tumor burden to understand why patients with high bTMB had worse prognosis. MAF was significantly lower in patients with low bTMB than those with high bTMB $(P<.001$; supplemental eFigure 10A). MAF correlated with baseline sum of the longest diameters (Spearman $\rho=0.175 ; P<.001$; supplemental eFigure 10B). 
A

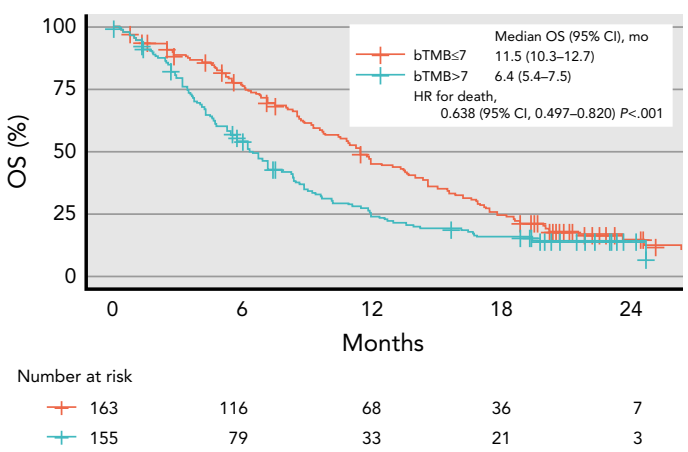

C

Subgroup

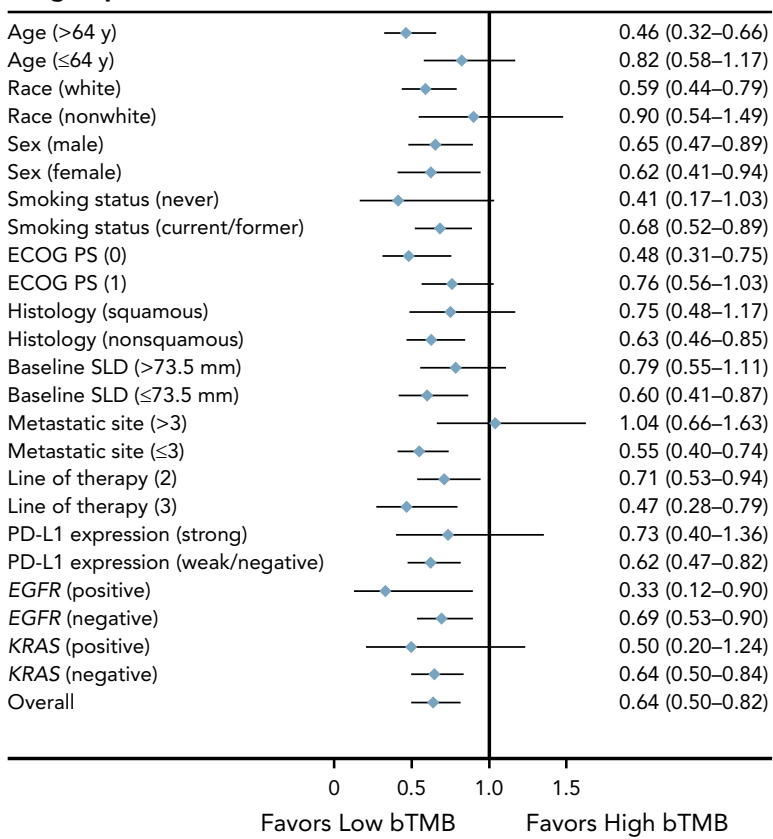

B

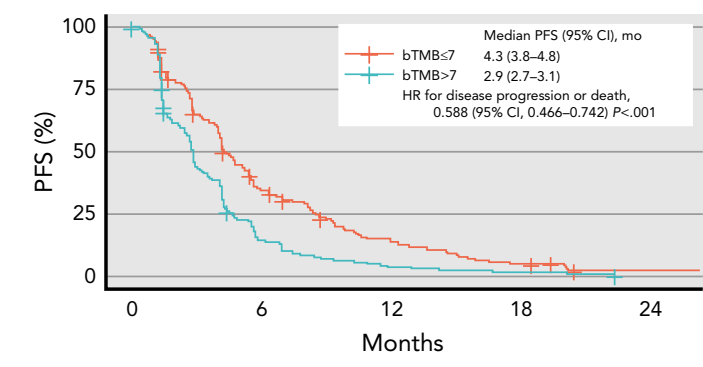

Number at risk
$+\quad 163$
$+\quad 155$

52
21

21

5

7
2

1

D

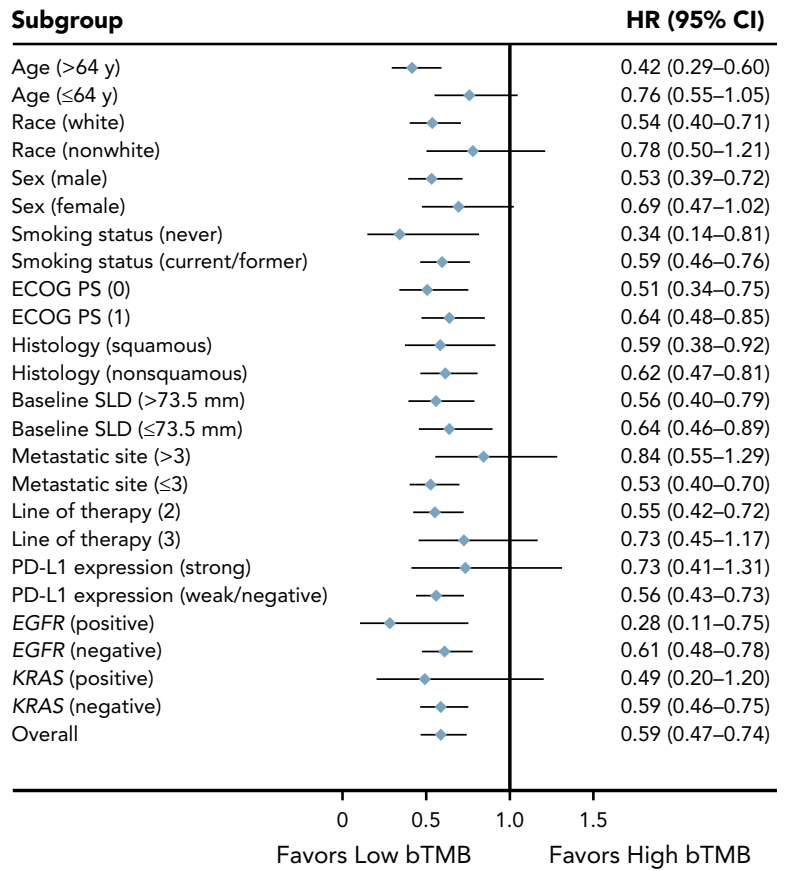

Figure 2. (A) Kaplan-Meier estimates of OS in low and high bTMB groups in the training set (OAK). (B) Kaplan-Meier estimates of PFS in low and high bTMB groups in the training set. (C) HR for OS in subgroups. (D) HR for PFS in subgroups.

Abbreviations: bTMB, blood-based tumor mutational burden; HR, hazard ratio; OS, overall survival; PFS, progression-free survival; PS, performance status; SLD, sum of the longest diameters.

Based on MAF above and below the median, higher MAF was associated with shorter OS (8.31 vs 9.63 months; HR, 1.387; 95\% CI, 1.119-1.719; $P=.003$; supplemental eFigure 10C), but not with PFS (2.99 vs 4.07 months; HR, 1.049 ; 95\% CI, $0.860-1.280 ; P=.636$; supplemental eFigure 10D). In addition, bTMB and baseline sum of longest diameters were significantly correlated (Spearman $\rho=0.349 ; P<.001$; supplemental eFigure 11).

\section{Discussion}

In this hypothesis-generating study, we found that bTMB was negatively associated with clinical benefit of docetaxel. To our knowledge, this is the first study in which patients with low bTMB had a demonstrated survival advantage when treated with docetaxel.

There are 2 potential explanations for why individuals with low bTMB may have longer PFS and OS. First, there was a significant positive correlation between bTMB and baseline sum of longest diameters and MAF. In this and previous studies, there was a significant correlation between tumor burden and MAF. ${ }^{16}$ Therefore, bTMB was associated with overall tumor burden and bTMB could reflect the general condition of patients with NSCLC, at least in part. Second, the elevated bTMB would correlate with more deleterious 
A

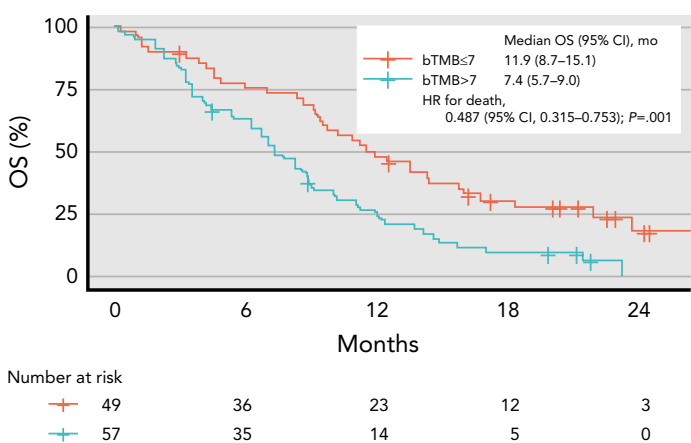

B

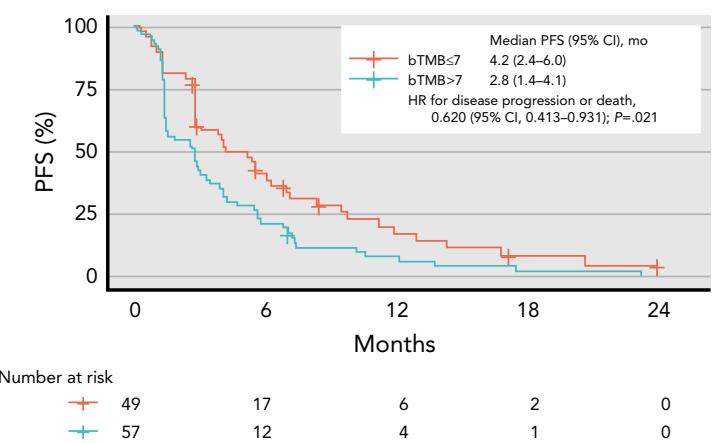

D

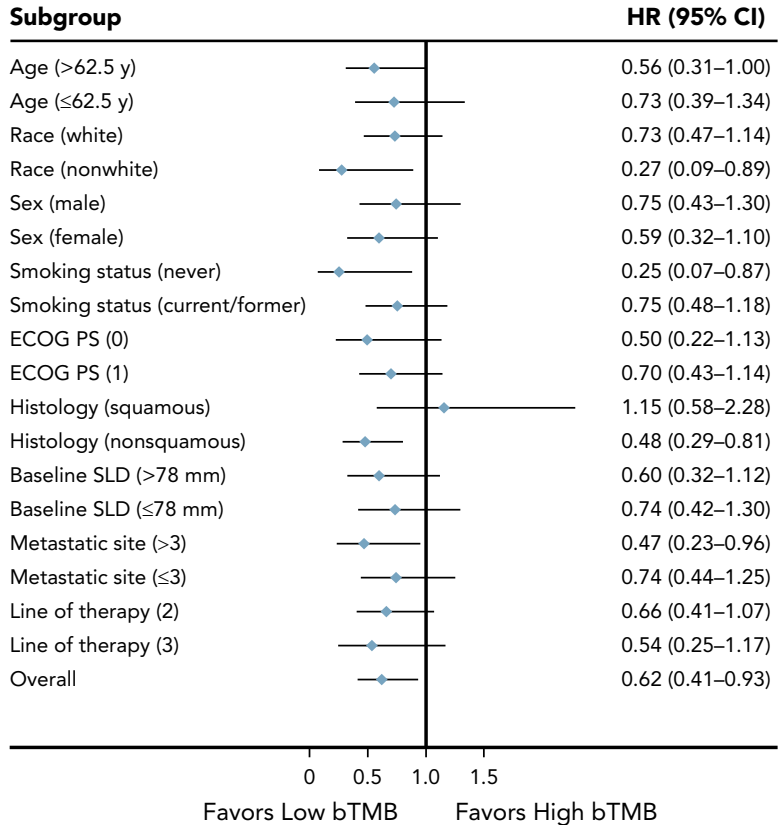

Figure 3. (A) Kaplan-Meier estimates of OS in low and high bTMB groups in the validation set. (B) Kaplan-Meier estimates of PFS in low and high bTMB groups in the validation set. (C) HR for OS in subgroups. (D) HR for PFS in subgroups.

Abbreviations: bTMB, blood-based tumor mutational burden; HR, hazard ratio; OS, overall survival; PD, progressive disease; PFS, progression-free survival; PS, performance status; SLD, sum of the longest diameters.

gene mutations, such as KEAP1, which could lead to clinical resistance.

Recently, Offin et $\mathrm{al}^{17}$ found a negative relation between TMB and outcomes with targeted therapy in patients with EGFR-mutant NSCLC. Patients with NSCLC and driver mutations had lower TMB than those without driver mutations. ${ }^{14}$ We speculated that these patients would derive benefit from EGFR TKIs combined with chemotherapy. Consistent with this hypothesis, we found that treatment with pemetrexed + carboplatin combined with gefitinib provided better survival benefits for patients with lung adenocarcinoma harboring sensitive EGFR mutations. ${ }^{18}$ Cheng et al ${ }^{19}$ also suggested that the addition of pemetrexed to gefitinib provided more clinical benefit than gefitinib monotherapy in patients with NSCLC with activating EGFR mutations. In addition, patients with ROS1/ALK rearrangement had a higher ORR and a longer PFS with pemetrexed than those without ROS1/ALK rearrangement. ${ }^{20-22}$ In Checkmate 026, PFS was significantly longer in the chemotherapy group than the nivolumab group among patients with NSCLC with low and medium TMB. ${ }^{23}$ Additionally, in the LACE-Bio-II study, Devarakonda et $\mathrm{al}^{24}$ found that lung cancerspecific survival benefit with adjuvant chemotherapy was more pronounced in patients with low TMB. Taken together, all of these studies suggest that patients with NSCLC with low TMB might derive a better prognosis from chemotherapy. 
Apart from bTMB, we also assessed the effect of specific gene alterations on benefit from chemotherapy. We found that mutations in KEAP1 were associated with lack of benefit. Singh et al ${ }^{25}$ suggested that inactivation of KEAPl was a frequent genetic alteration in NSCLC. Loss of KEAP1 function could lead to constitutive activation of Nrf2 and expression of cytoprotective genes, such as antioxidants, xenobiotic metabolism enzymes, and drug efflux pumps. ${ }^{25-27}$ Therefore, KEAP1 mutations could be the cause of tumor resistance against chemotherapeutic drugs.

This study had several limitations. First, this was a retrospective study, which was prone to bias. Therefore, the observed survival advantage in the low bTMB group may be a result of confounding, such as disease burden and higher frequency of low ECOG performance status. Although multivariate analysis and propensity score matching were conducted in our study, these methods could not remove confounding by factors not selected into the model. Second, other functional aberrations, such as hypermethylation and phosphorylation, may be critical to differential efficacy; however, blood-based NGS could not detect them. Third, tumors can acquire mutations as they evolve. Given that no postchemotherapy NGS data could be used, we were unable to determine the acquired resistance mechanisms. Fourth, a recent study found that a large proportion of mutations found in liquid biopsies do not come from tumor cells, but rather from alterations in white blood cells. ${ }^{28}$ In our study, the mutation frequency of DNMT3A seemed to be nearly $20 \%$ to $30 \%$, which was uncharacteristic for NSCLC. Thus, bTMB estimation may be likely to have been influenced by clonal hematopoietic variants in this analysis. Finally, we could not assess the association between bTMB and tissue TMB because of lack of data.

\section{Conclusions}

This study demonstrated that bTMB $(\leq 7 \mathrm{SNVs} / \mathrm{Mb})$ is prognostic and predictive of treatment outcome of docetaxel in patients with NSCLC. Prospective studies are needed to validate this result.

\section{Acknowledgments}

Data from the OAK and POPLAR trials included in this analysis were provided online by F. Hoffmann-La Roche. We would like to thank David S. Shames from Genentech and David R. Gandara from UC Davis Comprehensive Cancer Center, the lead authors of the $\mathrm{bTMB}$ primary manuscript, for making valuable comments on this manuscript. We also would like to thank Jia He and Yingyi Qin from the Department of Health Statistics, Second Military Medical University for their valuable comments on statistics analysis.

Submitted August 19, 2019; accepted for publication December 2, 2019

Author contributions: Literature search: Nie, J. Qian, Xu, Gu. Figures: Nie, J. Qian, Xu, Gu. Study design: Nie, J. Qian, Xu, Gu, Zhong, Han. Data collection: Nie, J. Qian, Xu, Gu, F.F. Qian, Lu, X.Y. Zhang, H.M. Wang, Yan, B. Zhang, S.Y. Wang, Hu, Li. Data analysis: Nie, J. Qian, Xu, Gu. Writing of manuscript: Nie, J. Qian, Xu, Gu, Zhong, Han. Review of manuscript: Zhong, Han.

Disclosures: Dr. Han has disclosed that he has received consultant fees from AstraZeneca and Roche Pharmaceutical Company and speaking fees from AstraZeneca Pharmaceutical Company and Lily Pharmaceutical Company. The remaining authors have disclosed that they have not received any financial consideration from any person or organization to support the preparation, analysis, results, or discussion of this article.

Funding: This study was sponsored by Shanghai Municipal Human Resource and Social Security Bureau Talent Project (No. 052), National Natural Science Foundation of China (No. 81601988, 81602078, and 81502450), Science and Technology Commission of Shanghai Municipality, China (No. 18441904700), and Shanghai Chest Hospital Project of Collaborative Innovation (No. YJXT20190102)

Disclaimer: Datasets analyzed during the current study are available from the corresponding author on reasonable request.

Correspondence: Hua Zhong, MD, Shanghai Chest Hospital, Shanghai Jiao Tong University, Huaihai West Road No. 241, Shanghai 200030, China. Email: eddiedong8@hotmail.com; and

Bao-Hui Han, MD, Shanghai Chest Hospital, Shanghai Jiao Tong University, Huaihai West Road No. 241, Shanghai 200030, China. Email: xkyyhan@gmail.com

\section{References}

1. Schiller JH, Harrington D, Belani CP, et al. Comparison of four chemotherapy regimens for advanced non-small-cell lung cancer. N Engl J Med 2002;346:92-98.

2. Scagliotti GV, De Marinis F, Rinaldi M, et al. Phase III randomized trial comparing three platinum-based doublets in advanced non-small-cell lung cancer. J Clin Oncol 2002;20:4285-4291.

3. Herbst RS, Baas P, Kim DW, et al. Pembrolizumab versus docetaxel for previously treated, PD-L1-positive, advanced non-small-cell lung cancer (KEYNOTE-010): a randomised controlled trial. Lancet 2016;387: 1540-1550.

4. Lee SM, Falzon M, Blackhall F, et al. Randomized prospective biomarker trial of ERCC1 for comparing platinum and nonplatinum therapy in advanced non-small-cell lung cancer: ERCC1 Trial (ET). J Clin Oncol 2017;35: 402-411.

5. Bepler G, Williams C, Schell MJ, et al. Randomized international phase III trial of ERCC1 and RRM1 expression-based chemotherapy versus gemcitabine/carboplatin in advanced non-small-cell lung cancer. J Clin Oncol 2013;31:2404-2412.

6. Seymour L, Le Teuff G, Brambilla E, et al. LACE-Bio: validation of predictive and/or prognostic immunohistochemistry/histochemistry-based biomarkers in resected non-small-cell lung cancer. Clin Lung Cancer 2019; 20:66-73.e6.

7. Chan TA, Yarchoan M, Jaffee $E$, et al. Development of tumor mutation burden as an immunotherapy biomarker: utility for the oncology clinic. Ann Oncol 2019;30:44-56.

8. Gandara DR, Paul SM, Kowanetz M, et al. Blood-based tumor mutationa burden as a predictor of clinical benefit in non-small-cell lung cancer patients treated with atezolizumab. Nat Med 2018;24:1441-1448.

9. Dagogo-Jack I, Shaw AT. Tumour heterogeneity and resistance to cancer therapies. Nat Rev Clin Oncol 2018;15:81-94.

10. Rittmeyer A, Barlesi F, Waterkamp D, et al. Atezolizumab versus docetaxe in patients with previously treated non-small-cell lung cancer (OAK): a phase 3, open-label, multicentre randomised controlled trial. Lancet 2017;389:255-265.

11. Fehrenbacher L, Spira A, Ballinger M, et al. Atezolizumab versus docetaxel for patients with previously treated non-small-cell lung cancer (POPLAR): a multicentre, open-label, phase 2 randomised controlled trial. Lancet 2016;387:1837-1846.

12. Frampton GM, Fichtenholtz A, Otto GA, et al. Development and validation of a clinical cancer genomic profiling test based on massively parallel DNA sequencing. Nat Biotechnol 2013;31:1023-1031.

13. Chalmers ZR, Connelly CF, Fabrizio D, et al. Analysis of 100,000 human cancer genomes reveals the landscape of tumor mutational burden. Genome Med 2017:9:34. 
14. Heagerty PJ, Lumley T, Pepe MS. Time-dependent ROC curves for censored survival data and a diagnostic marker. Biometrics 2000;56:337-344.

15. Lunt M. Selecting an appropriate caliper can be essential for achieving good balance with propensity score matching. Am J Epidemiol 2014;179: 226-235.

16. Chae $Y K$, Davis AA, Agte $S$, et al. Clinical implications of circulating tumor DNA tumor mutational burden (ctDNA TMB) in non-small cell lung cancer. Oncologist 2019;24:820-828

17. Offin M, Rizvi H, Tenet $M$, et al. Tumor mutation burden and efficacy of EGFR-tyrosine kinase inhibitors in patients with EGFR-mutant lung cancers. Clin Cancer Res 2019;25:1063-1069.

18. Han B, Jin B, Chu T, et al. Combination of chemotherapy and gefitinib as first-line treatment for patients with advanced lung adenocarcinoma and sensitive EGFR mutations: a randomized controlled trial. Int J Cancer 2017;141:1249-1256.

19. Cheng Y, Murakami H, Yang PC, et al. Randomized phase II trial of gefitinib with and without pemetrexed as first-line therapy in patients with advanced nonsquamous non-small-cell lung cancer with activating epidermal growth factor receptor mutations. J Clin Oncol 2016;34: 3258-3266.

20. Kim HR, Lim SM, Kim HJ, et al. The frequency and impact of ROS1 rearrangement on clinical outcomes in never smokers with lung adenocarcinoma. Ann Oncol 2013:24:2364-2370.
21. Chen YF, Hsieh MS, Wu SG, et al. Efficacy of pemetrexed-based chemotherapy in patients with ROS1 fusion-positive lung adenocarcinoma compared with in patients harboring other driver mutations in East Asian populations. J Thorac Oncol 2016;11:1140-1152.

22. Camidge DR, Kono SA, Lu X, et al. Anaplastic lymphoma kinase gene rearrangements in non-small cell lung cancer are associated with prolonged progression-free survival on pemetrexed. J Thorac Oncol 2011;6:774-780.

23. Carbone DP, Reck M, Paz-Ares $L$, et al. First-line nivolumab in stage IV or recurrent non-small-cell lung cancer. N Engl J Med 2017;376:2415-2426.

24. Devarakonda S, Rotolo F, Tsao MS, et al. Tumor mutation burden as a biomarker in resected non-small-cell lung cancer. J Clin Oncol 2018;36: 2995-3006

25. Singh A, Misra V, Thimmulappa RK, et al. Dysfunctional KEAP1-NRF2 interaction in non-small-cell lung cancer. PLoS Med 2006;3:e420.

26. Shibata T, Kokubu A, Gotoh M, et al. Genetic alteration of Keap1 confers constitutive Nrf2 activation and resistance to chemotherapy in gallbladder cancer. Gastroenterology 2008;135:1358-1368.

27. Ohta T, lijima K, Miyamoto M, et al. Loss of Keap1 function activates Nrf2 and provides advantages for lung cancer cell growth. Cancer Res 2008;68: 1303-1309.

28. Razavi P, Li BT, Brown DN, et al. High-intensity sequencing reveals the sources of plasma circulating cell-free DNA variants. Nat Med 2019;25 1928-1937.

See JNCCN.org for supplemental online content.

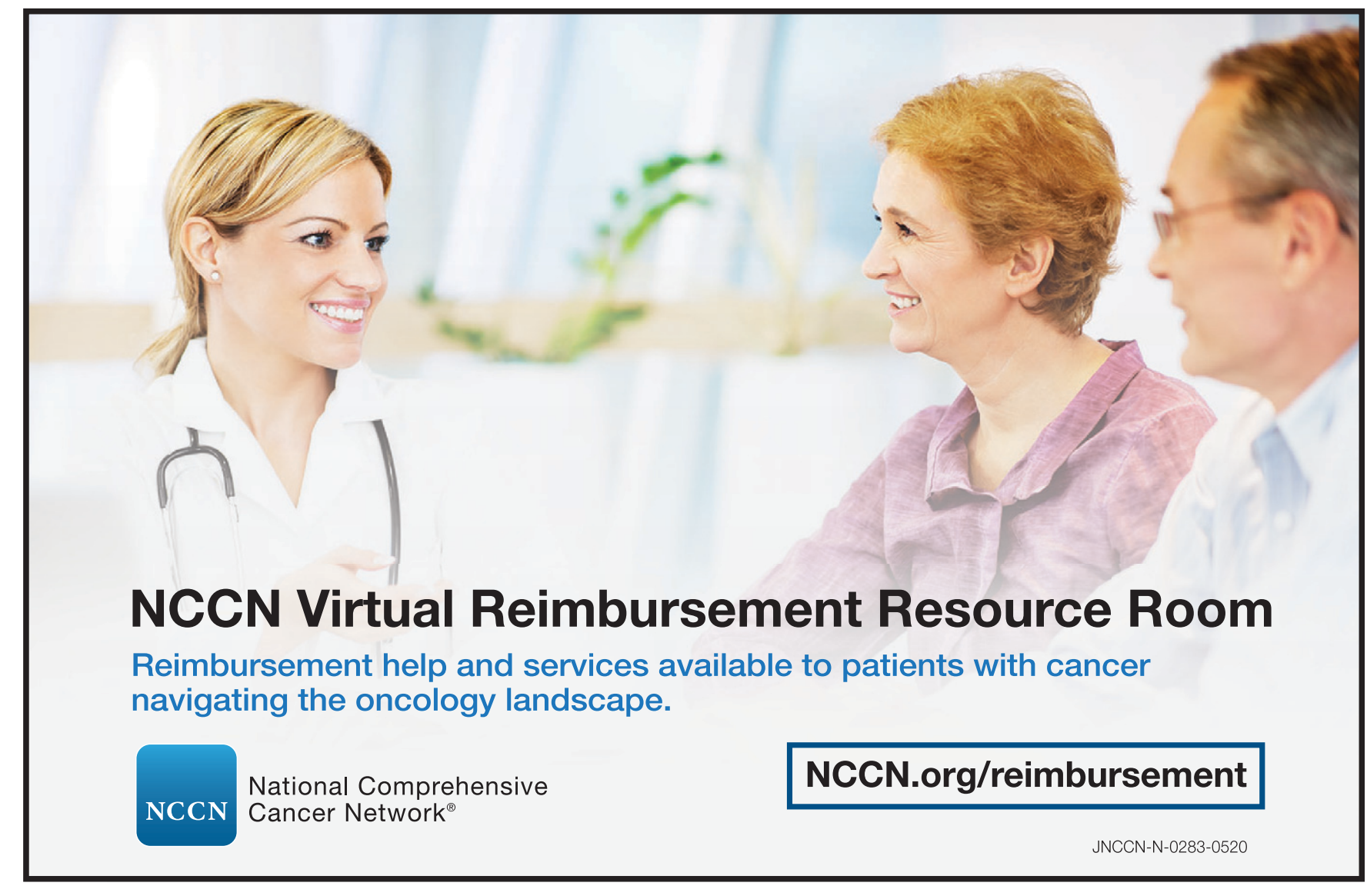


Supplemental online content for:

\section{Prognostic and Predictive Value of Blood Tumor Mutational Burden in Patients With Lung Cancer Treated With Docetaxel}

Wei Nie, MD; Jie Qian, MD; Mi-Die Xu, MD; Kai Gu, MD; Fang-Fei Qian, MD; Jun Lu, MD; Xue-Yan Zhang, MD; Hui-Min Wang, MD; Bo Yan, MD; Bo Zhang, MD; Shu-Yuan Wang, MD; Fang Hu, MD; Chang-Hui Li, MD;

Hua Zhong, MD; and Bao-Hui Han, MD

J Natl Compr Canc Netw 2020;18(5):582-589

eFigure 1: Process of Patient Selection in the Training and Validation Sets

eFigure 2: Proportion of DCB Above or Below Percentiles of bTMB

eFigure 3: Proportion of Patients with PR, SD, and PD Below and Above the bTMB Cutoff Point of 7 SNVs/Mb in the Training, Validation, and Combination Sets

eFigure 4: Kaplan-Meier estimates of OS and PFS in Low and High bTMB Groups in Propensity-Matched Population eFigure 5: bTMB, OncoPrint, and Clinical Factors for Patients in Low and High bTMB Groups

eFigure 6: OncoPrint for Patients in PR, SD, and PD Groups

eFigure 7: OncoPrint of the Deleterious Genes Associated With Short Progression-Free and Overall Survivals in Low and High bTMB Groups

eFigure 8: OncoPrint for patients in NDB and DCB Groups

eFigure 9: KEAP1 Mutations

eFigure 10: MAF in Patients With High and Low bTMB

eFigure 11: Scatterplot of bTMB Versus Baseline Sum of Longest Diameters in Individual Tumors

eTable 1: Patient Characteristics

eTable 2: Characteristics of Patients in Training Set by bTMB Level

eTable 3: Prognostic Value of bTMB for PFS and OS in Training Set

eTable 4: Characteristics of Patients in Validation Set by bTMB Level

eTable 5: Prognostic Value of bTMB for PFS and OS in Validation Set

eTable 6: Patient Characteristics Before and After Propensity Score Matching

eTable 7: Doubly Robust Estimation for PFS and OS in Propensity Score-Matched Set

eTable 8: Treatment Interaction With bTMB in the Combined Set 

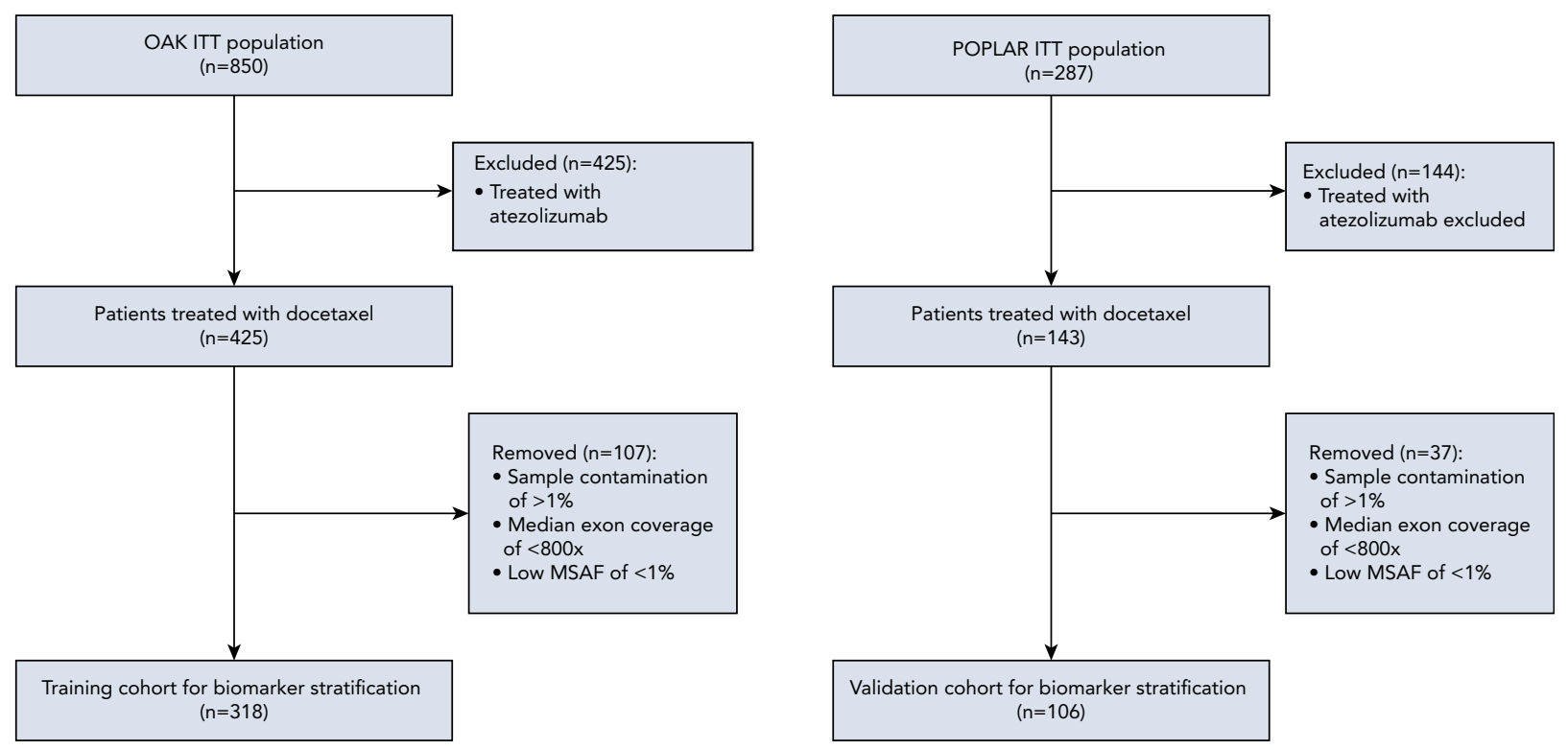

eFigure 1. Process of patient selection in the training (OAK cohort) and validation (POPLAR cohort) sets.

Abbreviations: ITT, intention to treat; MSAF, maximum somatic allele frequency. 
A

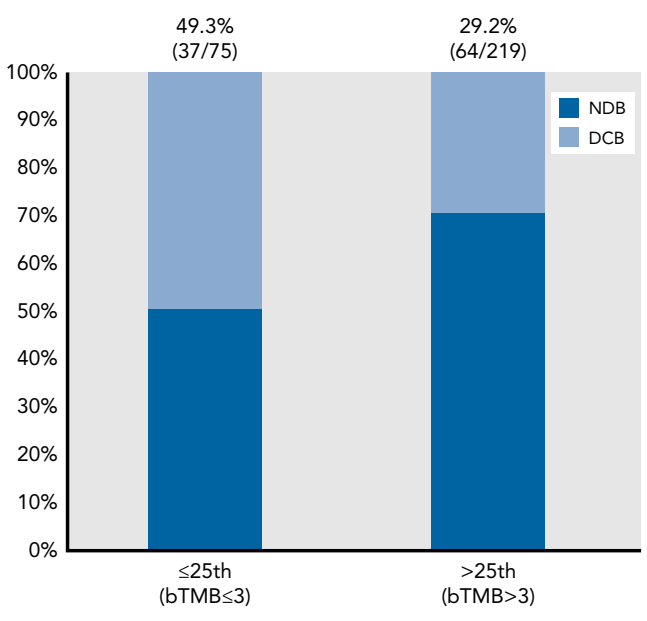

C

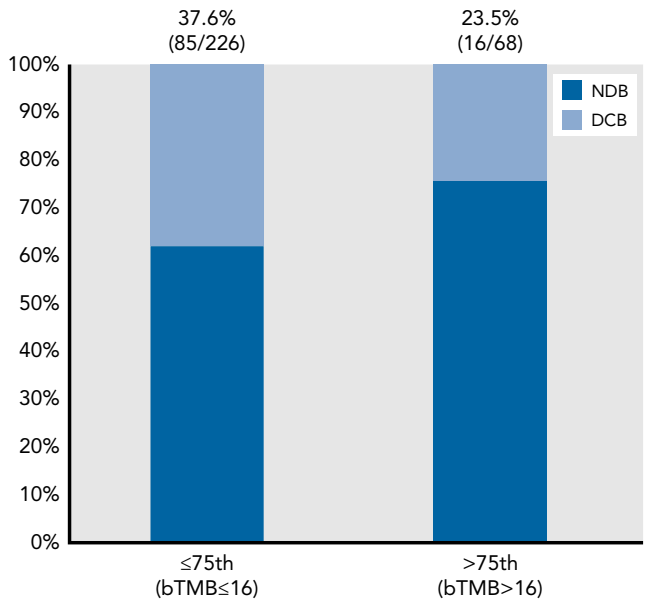

B

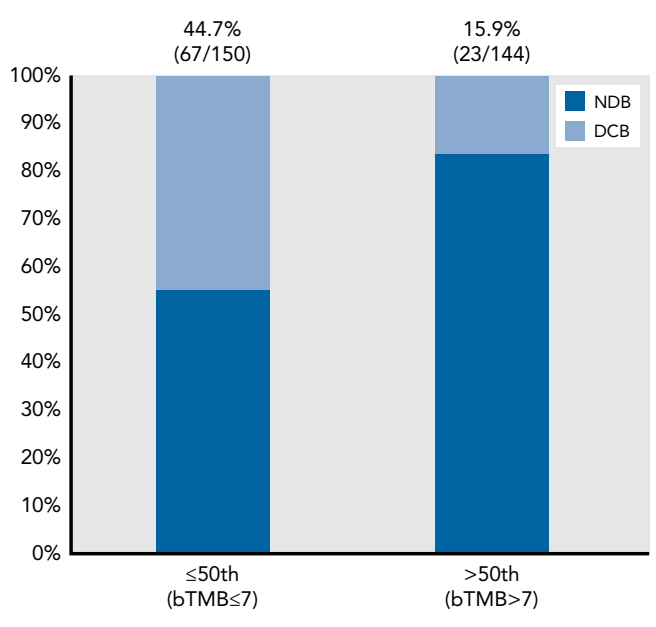

D

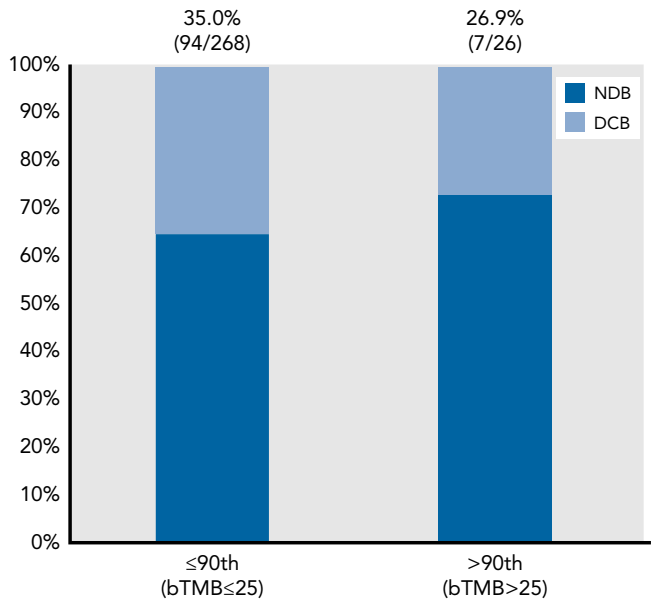

eFigure 2. Proportion of DCB above or below the (A) 25th, (B) 50th, (C) 75th, and (D) 90th percentiles of bTMB. Abbreviations: bTMB, blood-based tumor mutational burden; DCB, durable clinical benefit; NDB, no durable response. 
Nie et al - 3

A

OAK

ORR: $\quad 17.1 \%(25 / 146) \quad 11.7 \%(15 / 128)$

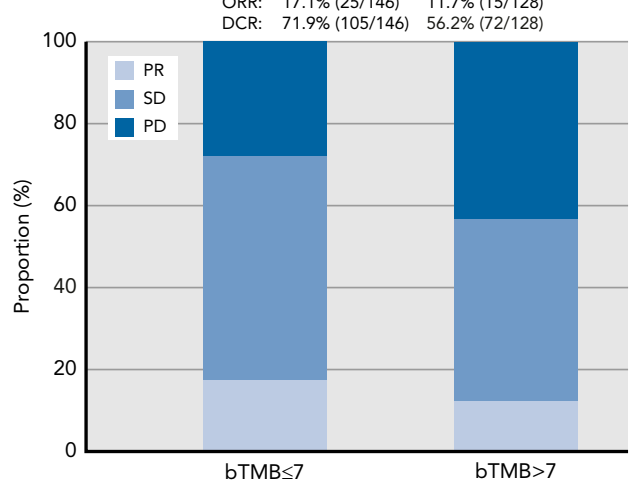

B

POPLAR

OAK + POPLAR
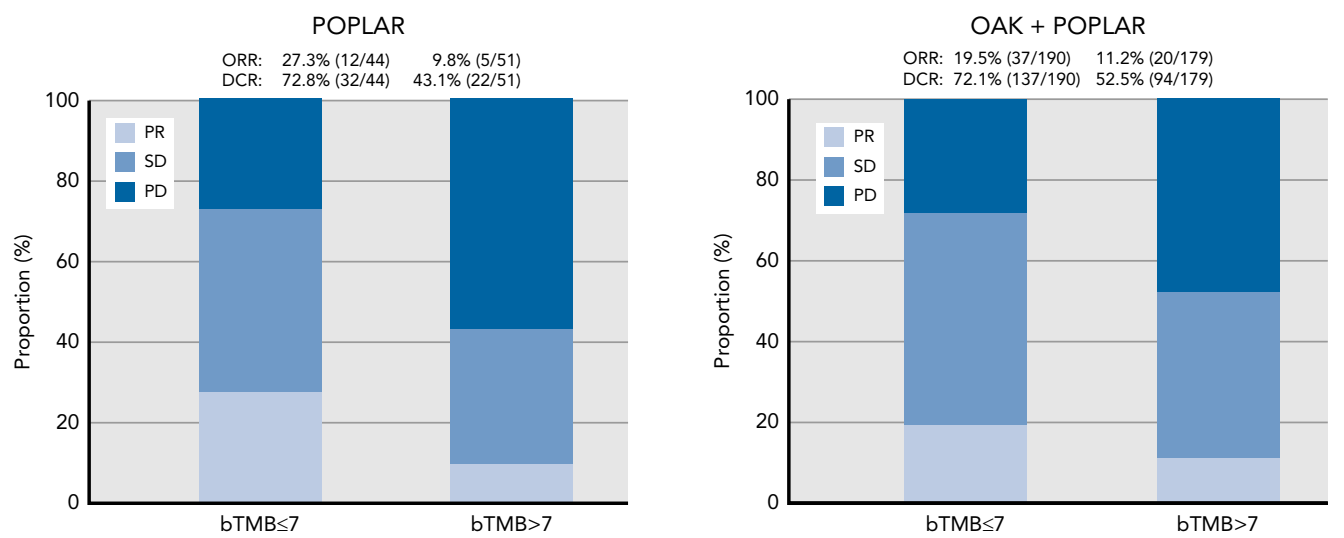

eFigure 3. Proportion of patients with $P R, S D$, and PD below and above the bTMB cutoff point of $7 \mathrm{SNV}$ s/Mb in the (A) training (OAK cohort), (B) validation (POPLAR cohort), and (B) combination sets (OAK + POPLAR).

Abbreviations: bTMB, blood-based tumor mutational burden; $D C R$, disease control rate; ORR, objective response rate; $P D$, progressive disease; PR, partial response; $\mathrm{SD}$, stable disease. 
A

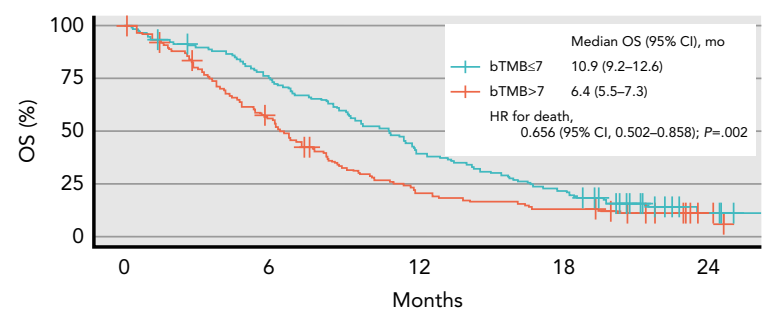

Number at risk

$\begin{array}{rrrrr}+\quad 129 & 67 & 24 & 15 & 3 \\ +\quad 129 & 95 & 52 & 27 & 4\end{array}$

B

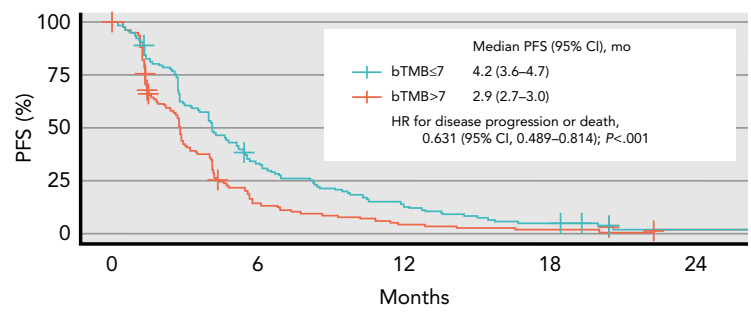

Number at risk

$\begin{array}{rrrrr}+\quad 129 & 17 & 5 & 2 & 0 \\ +\quad 129 & 42 & 18 & 6 & 1\end{array}$

eFigure 4. Kaplan-Meier estimates of (A) OS and (B) PFS in low and high bTMB groups in propensity-matched population. Abbreviations: bTMB, blood-based tumor mutational burden; HR, hazard ratio; OS, overall survival; PFS, progression-free survival. 
A

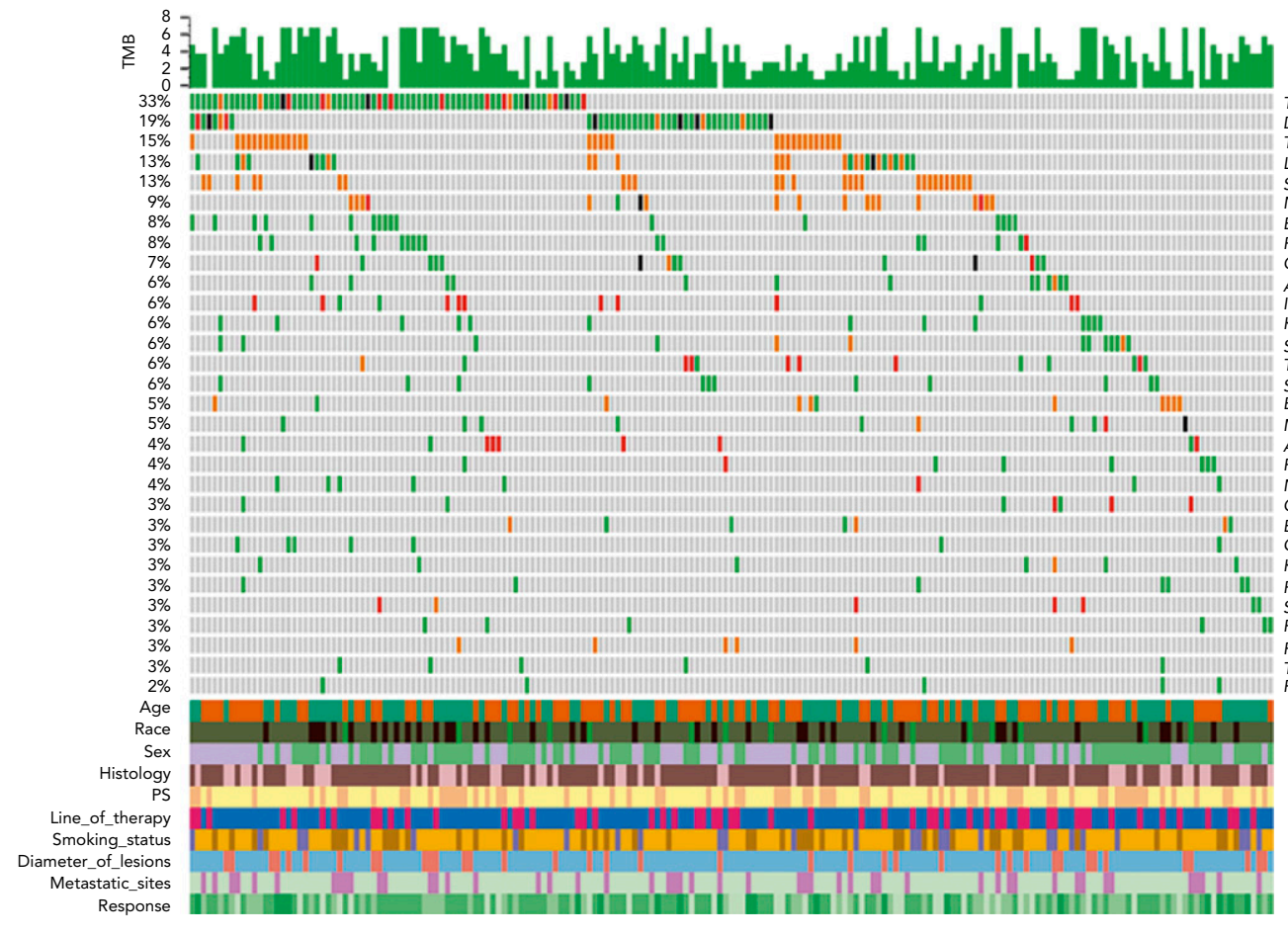

B

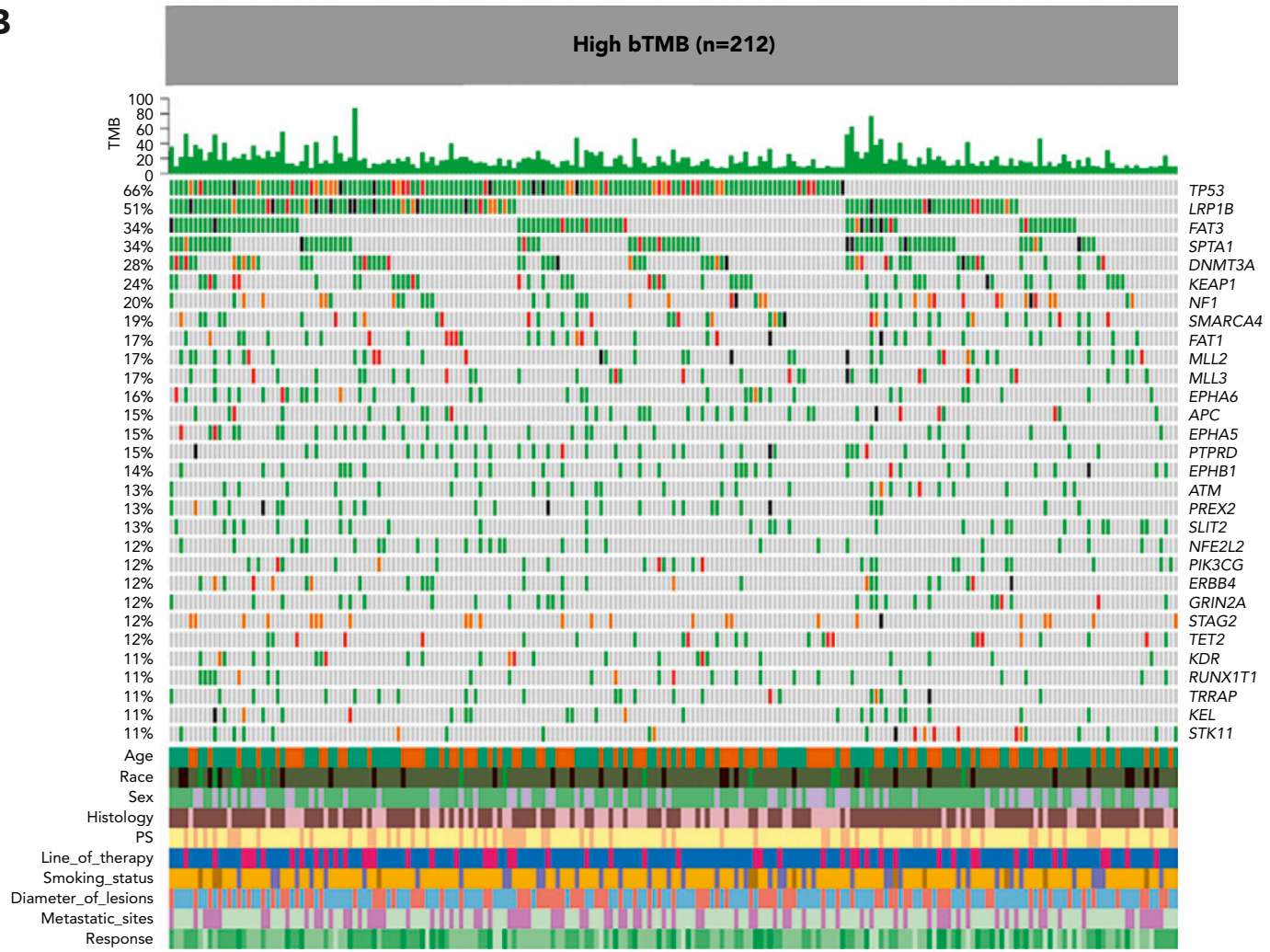

Age

$<65 \mathrm{y}$
$265 \mathrm{y}$

Race

White

Asian

Other

Sex

Male

Female

Histology

Nonsquamous

Squamous

PS

0

1

Second

Third

Smoking_status

Current

Previous

Never

Diameter_of_lesions

- $\leq 100 \mathrm{~mm}$

- >100 mm

Metastatic_sites

$\leq 3$

- $>3$

Response

PD

SD

Not evaluable

Genetic alteration

Missense mutation

Splice site

Nonsense mutation

Multi hit

eFigure 5. bTMB, OncoPrint, and clinical factors for patients in (A) low and (B) high bTMB groups.

Abbreviations: $\mathrm{bTMB}$, blood-based tumor mutational burden; PD, progressive disease; PR, partial response; PS, performance status; $S D$, stable disease; TMB, tumor mutational burden. 
A

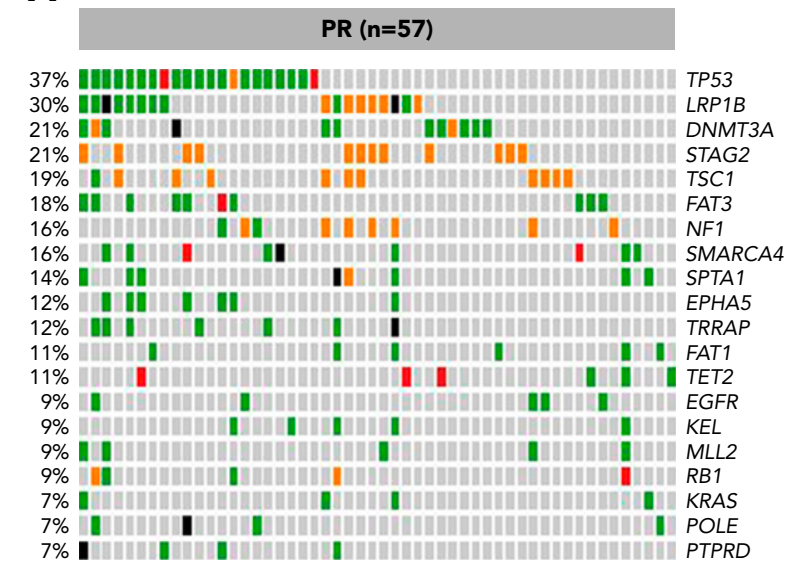

B

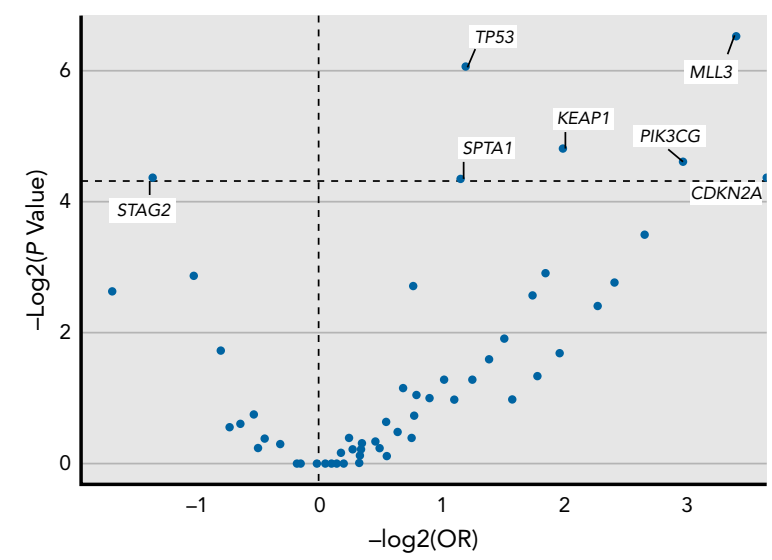

C

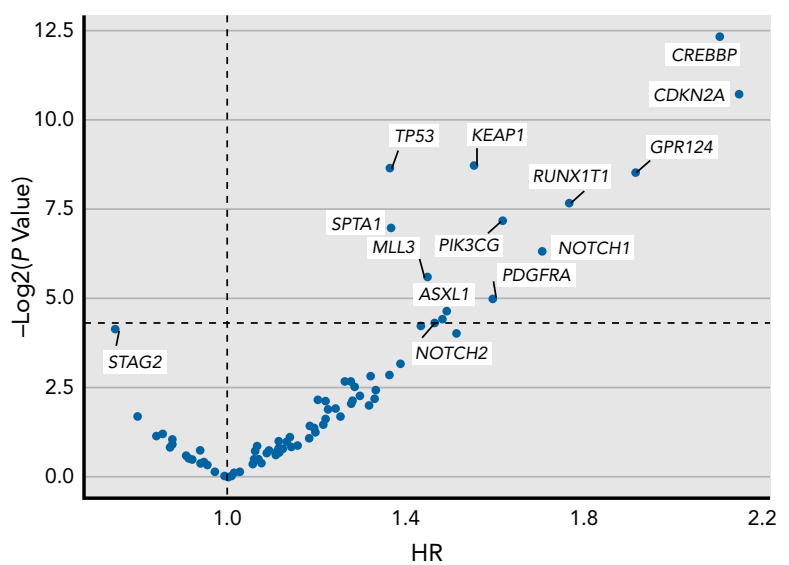

D

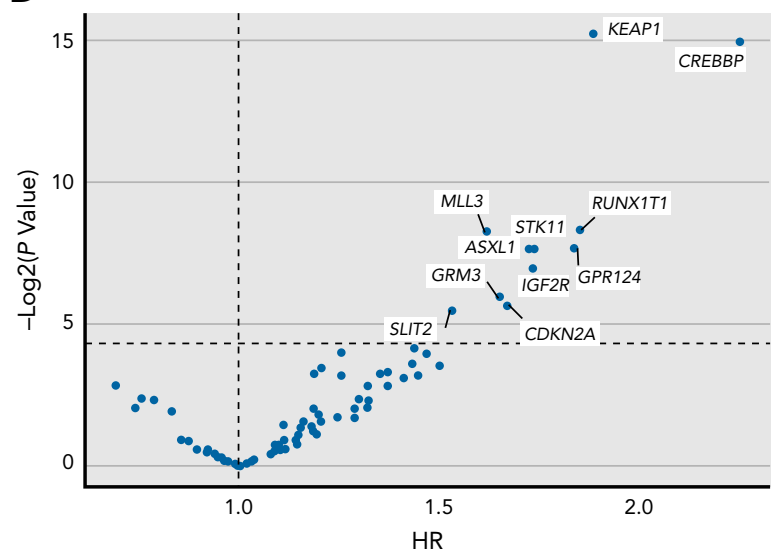

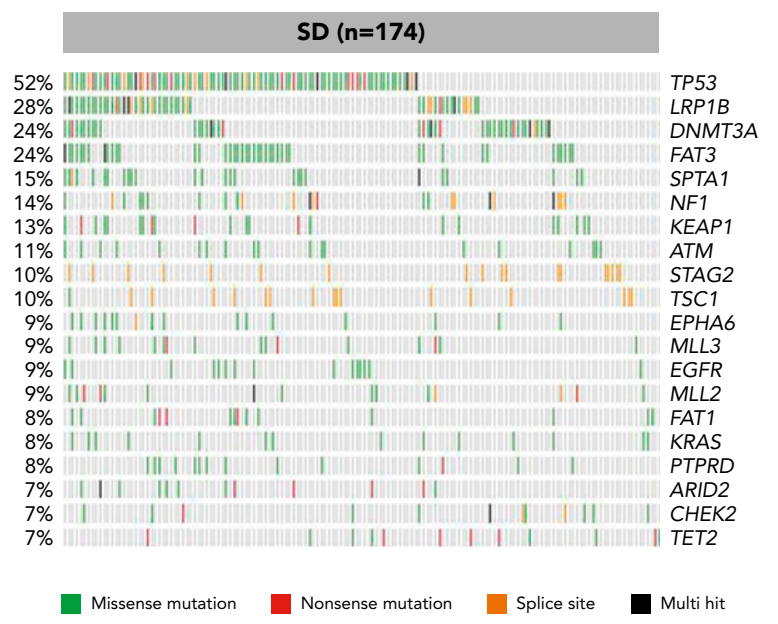

eFigure 6. (A) OncoPrint for patients in PR, SD, and PD groups. Log2 (OR) and - $\log 2(P$ value) for enrichment of individual altered genes in group comparisons of (B) PR versus PD. HRs and -log2(P value) for enrichment of individual altered genes associated with (C) PFS and (D) OR. Abbreviations: $\mathrm{HR}$, hazard ratio; $\mathrm{OR}$, odds ratio; $\mathrm{OS}$, overall survival; $\mathrm{PD}$, progressive disease; $\mathrm{PR}$, partial response; $\mathrm{PFS}$, progression-free survival; $\mathrm{SD}$, stable disease. 


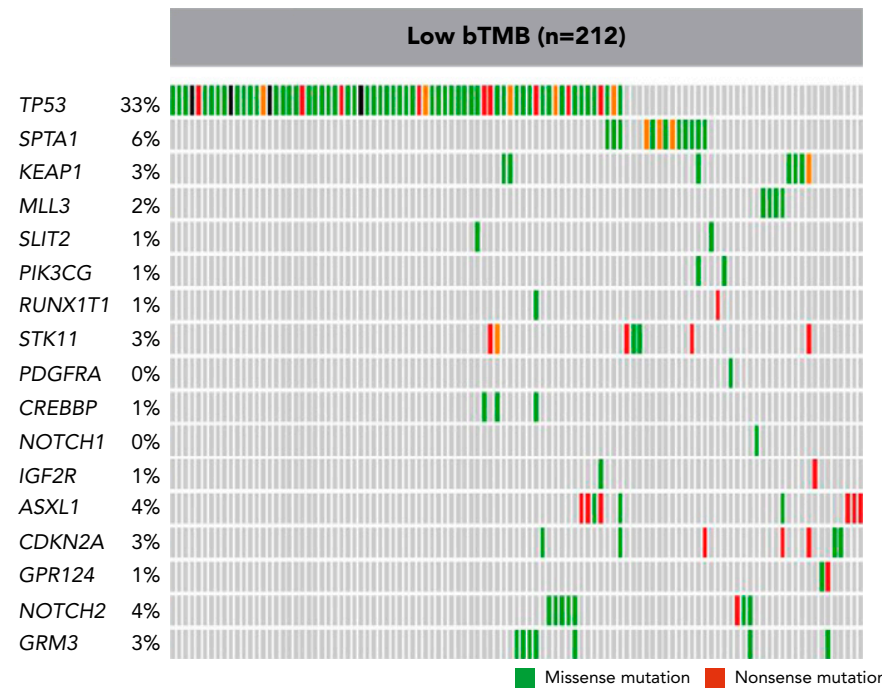

\section{High bTMB ( $=212)$}

66\% | |||||||||||||||||||||||||||||||||||||||||||||||||||||||||||||||||||||||||

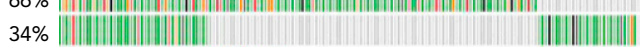

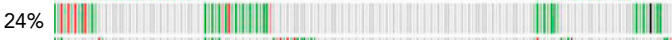
17\% || || || || ||| || ||||||| || || || || || ||| || || || 13\% || || || || || || || || || || || || || || || || || || || || || || || || ||

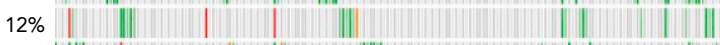

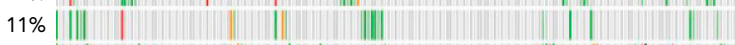
$11 \%$ | | | | || || || || || || || || || || || || || || || || || $10 \%$ | || || || ||| || || || || || || || || || || || || || || ||

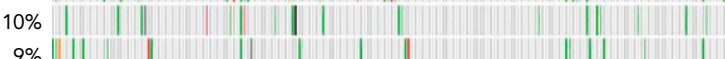
$9 \%$
$9 \%$

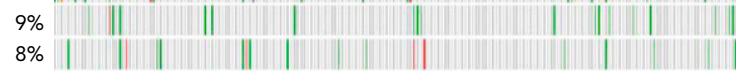

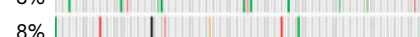
7\%

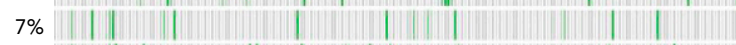
$6 \%$

Splice site

Multi hit

eFigure 7. OncoPrint of the deleterious genes associated with short progression-free and overall survivals in low and high bTMB groups. Abbreviation: bTMB, blood-based tumor mutational burden. 
A

NDB $(n=269)$

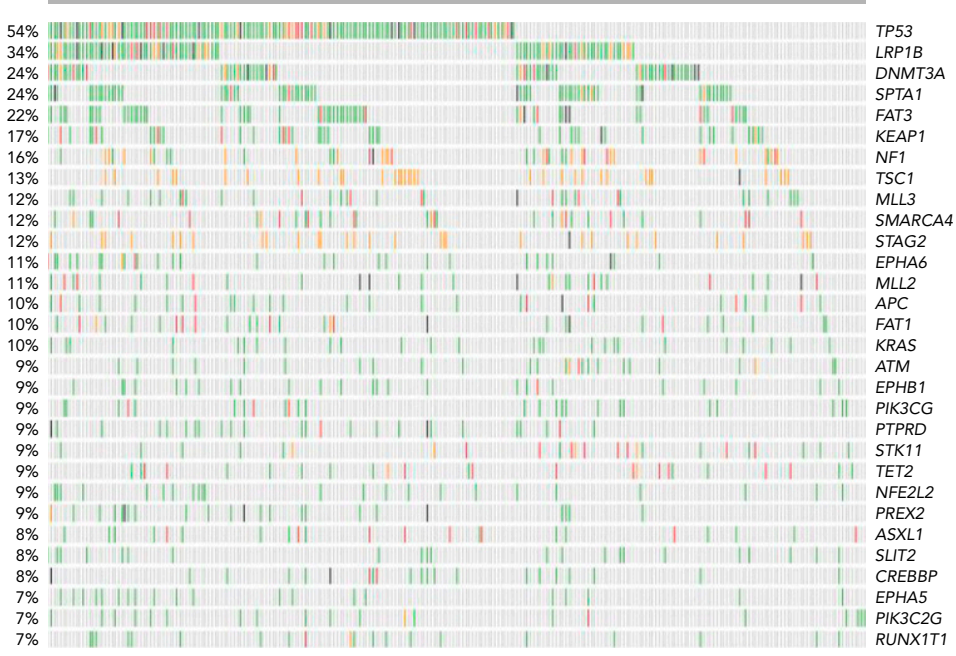

B

$\mathrm{DCB}(\mathrm{n}=138)$

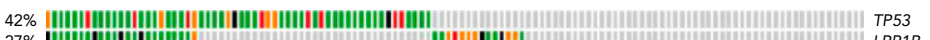
27\% $23 \%$
$19 \%$
IIIII $19 \%$. $19 \%$

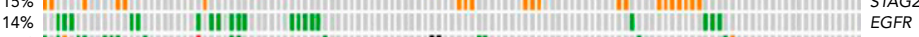

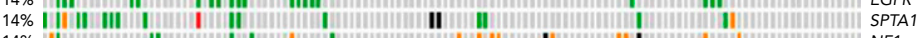
14\%

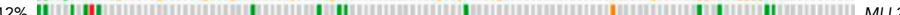
$12 \%$ $9 \%$ $9 \%$. $9 \%$ $9 \%$ $9 \%$

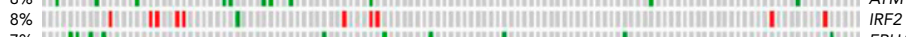

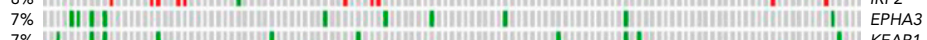
7\% $7 \%$ $7 \%$.1 1 $7 \%$ ㄴ. 1 7\% :ІІ І $7 \%$.

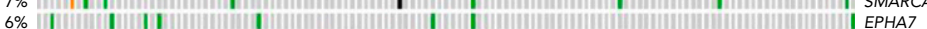

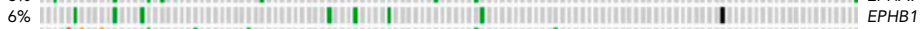

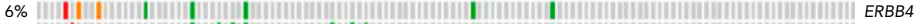

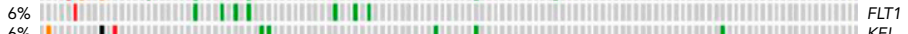

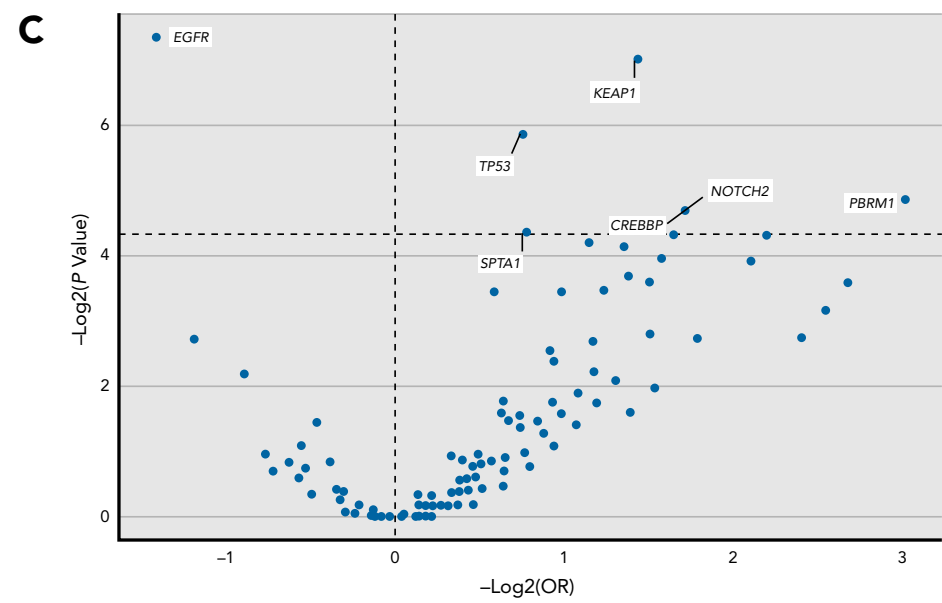

eFigure 8. OncoPrint for patients in (A) NDB and (B) DCB. (C) Log2(OR) and-log2(P value) for enrichment of individual altered genes in group comparisons of NDB and DCB.

Abbreviations: DCB, durable clinical benefit; NDB, no durable benefit; OR, odds ratio. 
A
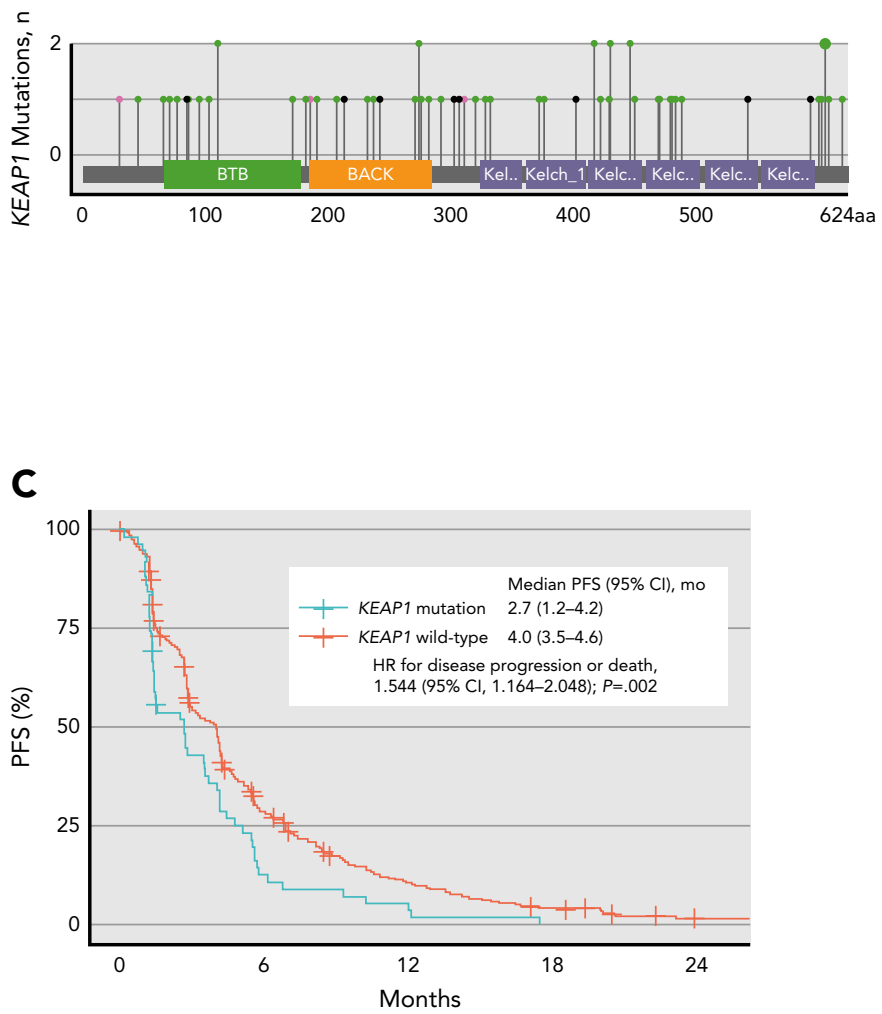

Number at risk

$+\quad 365$
$+\quad 59$
33

3
B

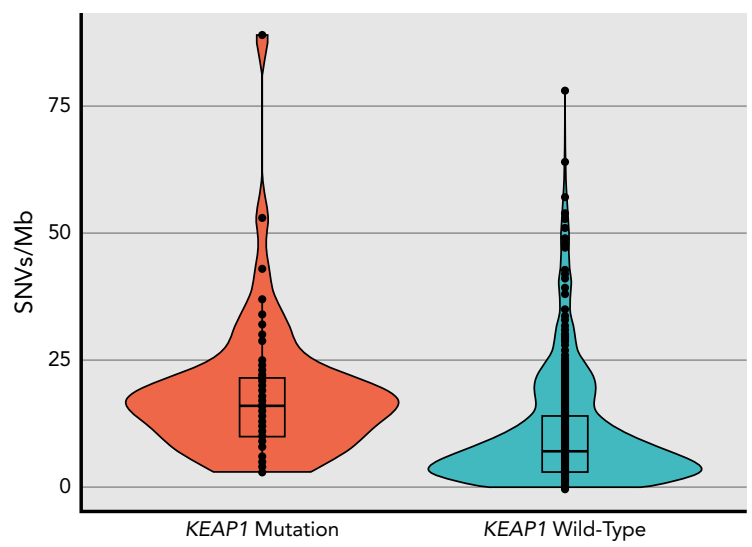

D

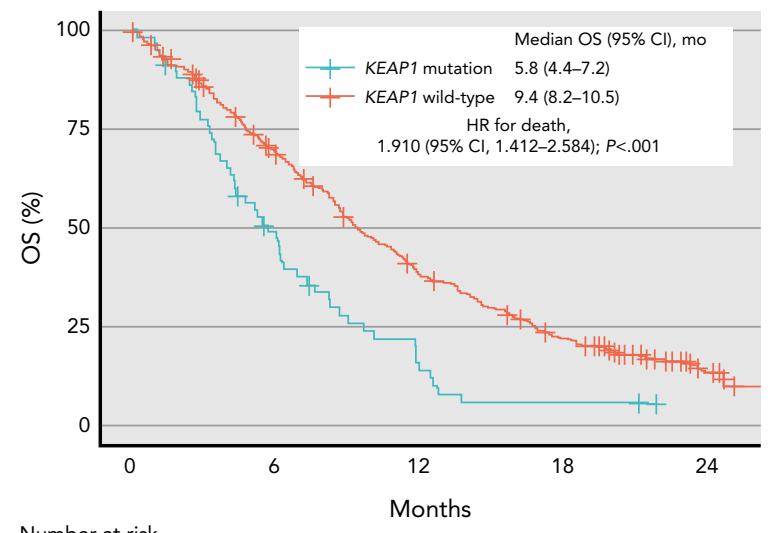

Number at risk

$\begin{array}{rrrrr}+\quad 365 & 240 & 128 & 71 & 13 \\ +\quad 59 & 26 & 10 & 3 & 0\end{array}$

eFigure 9. (A) Distribution of mutations in KEAP1. (B) BTB in patients with and without KEAP1 mutations. Kaplan-Meier estimates of (C) PFS and (D) OS in patients with and without KEAP1 mutations.

Abbreviations: BACK, BTB and C-terminal Kelch; BTB, broad-complex, Tramtrack, and Bric-a-brac; HR, hazard ratio; Mb, megabase; OS, overall survival; PFS, progression-free survival. 
A

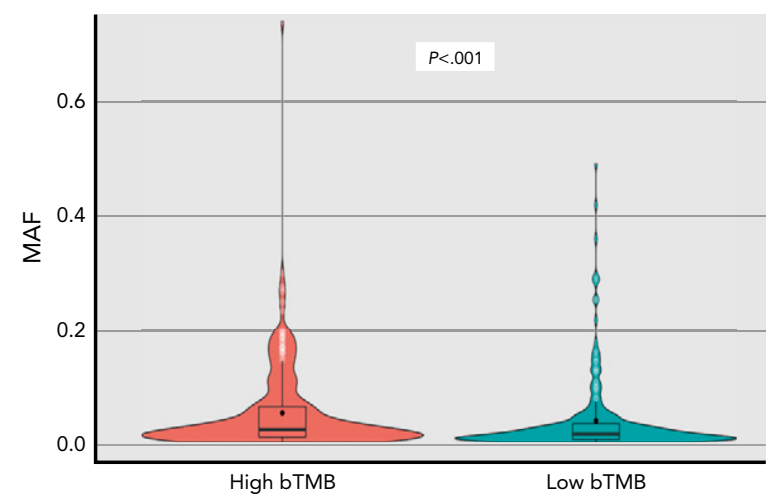

C

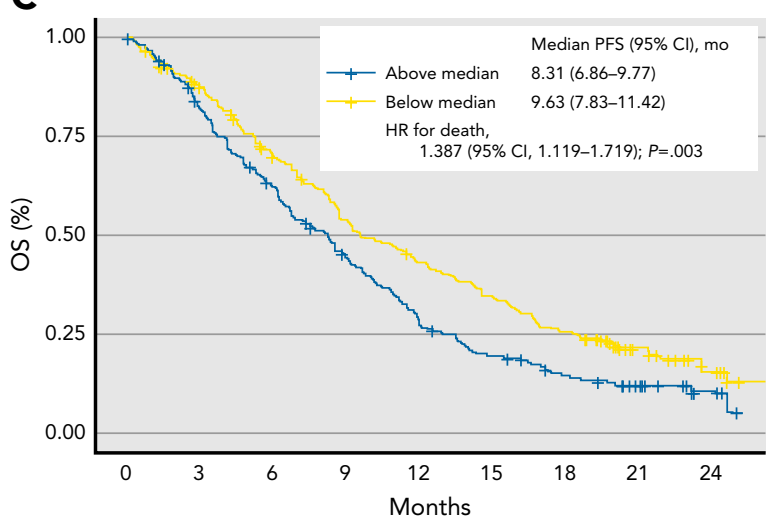

Number at risk

$\begin{array}{rrrrrrrrr}+212 & 179 & 139 & 104 & 82 & 66 & 49 & 25 & 9 \\ +212 & 170 & 127 & 87 & 56 & 37 & 25 & 15 & 4\end{array}$

\section{B}

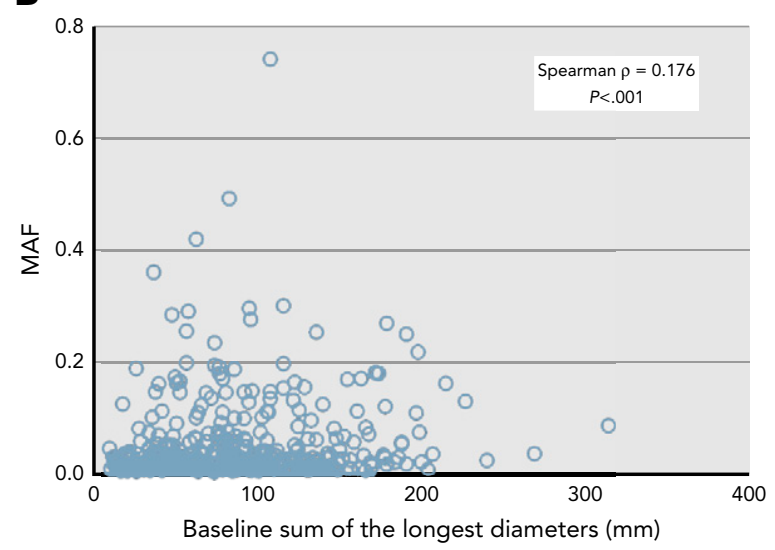

D

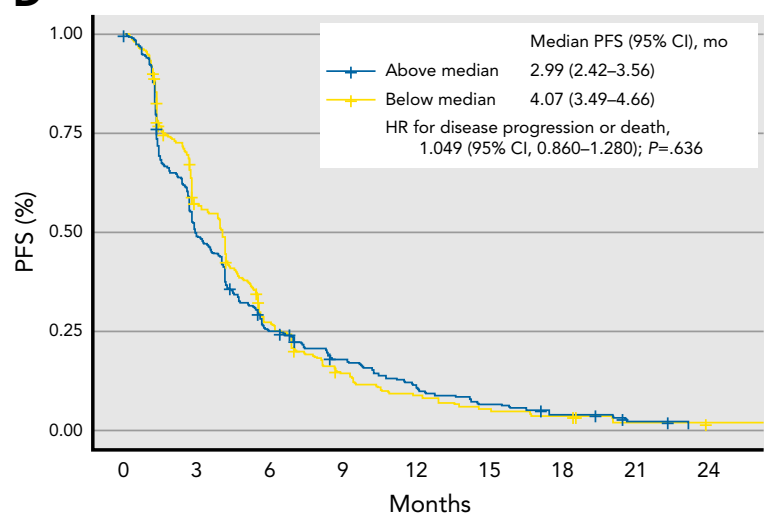

Number at risk

$\begin{array}{rrrrrrrrr}+212 & 113 & 51 & 25 & 15 & 9 & 6 & 2 & 1 \\ +212 & 104 & 51 & 33 & 21 & 12 & 6 & 2 & 0\end{array}$

eFigure 10. (A) MAF in patients with high and low bTMB. (B) Scatterplot of MAF versus baseline sum of longest diameters in individual tumors. Kaplan-Meier estimates of (C) OS and (D) PFS in patients based on MAF.

Abbreviations: bTMB, blood-based tumor mutational burden; MAF, median allele frequency; OS, overall survival; PFS, progression-free survival. 


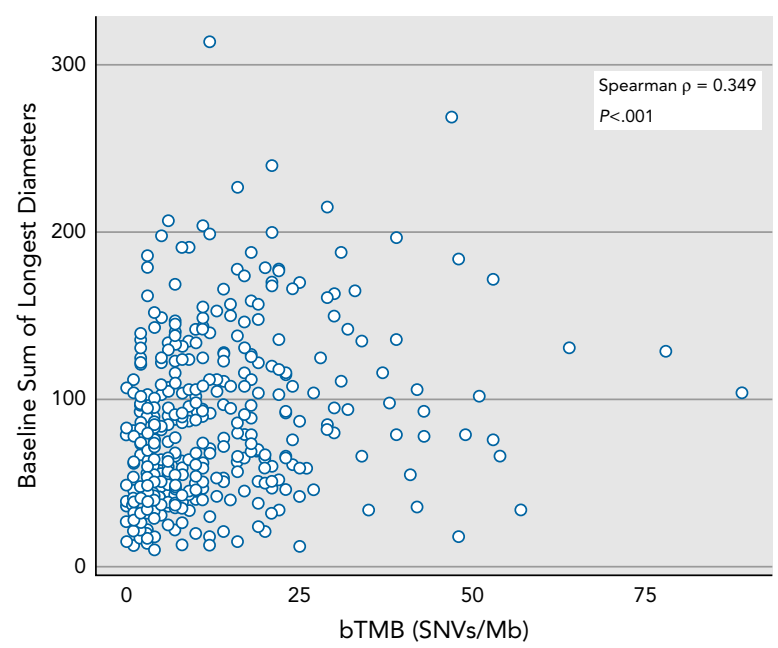

eFigure 11. Scatterplot of bTMB versus baseline sum of longest diameters in individual tumors. Abbreviations: bTMB, blood-based tumor mutational burden; SNVs/Mb, single-nucleotide variants/megabase. 


\section{eTable 1. Patient Characteristics}

\begin{tabular}{|c|c|c|c|}
\hline Characteristic & $\begin{array}{c}\text { Training Set (OAK) } \\
\mathbf{n}(\%)\end{array}$ & $\begin{array}{c}\text { Validation Set (POPLAR) } \\
n(\%)\end{array}$ & $P$ Value \\
\hline Total, $\mathrm{n}$ & 318 & 106 & \\
\hline Age, median (range), y & $64(34-85)$ & $62.5(36-80)$ & .170 \\
\hline Race & & & .005 \\
\hline White & $223(70.1)$ & $89(84.0)$ & \\
\hline Nonwhite ${ }^{a}$ & 95 (29.9) & $17(16.0)$ & \\
\hline Sex (\% female) & $124(39.0)$ & $48(45.3)$ & .253 \\
\hline Smoking status & & & .478 \\
\hline Never & 45 (14.2) & $18(17.0)$ & \\
\hline Former/Current & $273(85.8)$ & $88(83.0)$ & \\
\hline ECOG performance status & & & .211 \\
\hline 0 & $111(34.9)$ & $30(28.3)$ & \\
\hline 1 & $207(65.1)$ & $76(71.7)$ & \\
\hline Histology & & & .181 \\
\hline Nonsquamous & $226(71.1)$ & $68(64.2)$ & \\
\hline Squamous & 92 (28.9) & $38(35.8)$ & \\
\hline Line of therapy (\% 2 lines) & $236(74.2)$ & $76(71.7)$ & 611 \\
\hline Baseline SLD, median (range), mm & $73.5(10-314)$ & $78(11-269)$ & .197 \\
\hline Number of metastatic sites, median (IQR) & $3(2-4)$ & $3(2-4)$ & .115 \\
\hline PD-L1 (\% positive) & $169(53.7)$ & NA & NA \\
\hline PD-L1 (\% strong) & $52(16.5)$ & NA & NA \\
\hline EGFR mutation & $28(10.8)$ & $5(4.7)$ & .174 \\
\hline KRAS mutation & $25(7.9)$ & $10(9.4)$ & .610 \\
\hline
\end{tabular}

Abbreviations: IQR, interquartile range; NA, not available; SLD, sum of the longest diameters. alncluding Asian, black, and others. 


\section{eTable 2. Characteristics of Patients in Training Set by bTMB Level}

\begin{tabular}{|c|c|c|c|c|}
\hline Characteristic & $\begin{array}{l}\text { All Patients } \\
\text { n (\%) }\end{array}$ & $\begin{array}{c}\text { Low bTMB (TMB } \leq 7) \\
\text { n (\%) }\end{array}$ & $\begin{array}{c}\text { High bTMB (bTMB >7) } \\
\text { n (\%) }\end{array}$ & $P$ Value \\
\hline Total, $n$ & 318 & 163 & 155 & \\
\hline Age, median (range), y & $64(34-85)$ & $64(34-85)$ & $64(40-84)$ & .631 \\
\hline Race & & & & .023 \\
\hline White & $223(70.1)$ & $105(64.4)$ & $118(76.1)$ & \\
\hline Nonwhite ${ }^{a}$ & 95 (29.9) & $58(35.6)$ & 37 (23.9) & \\
\hline Sex (\% female) & $124(39.0)$ & $75(46.0)$ & 49 (31.6) & .009 \\
\hline Smoking status & & & & $<.001$ \\
\hline Never & 45 (14.2) & $38(23.3)$ & $7(4.5)$ & \\
\hline Former/Current & $273(85.8)$ & $125(76.7)$ & $148(95.5)$ & \\
\hline ECOG performance status & & & & .465 \\
\hline 0 & $111(34.9)$ & $60(36.8)$ & 51 (32.9) & \\
\hline 1 & $207(65.1)$ & $103(63.2)$ & $104(67.1)$ & \\
\hline Histology & & & & .023 \\
\hline Nonsquamous & $226(71.1)$ & $125(76.7)$ & $101(65.2)$ & \\
\hline Squamous & 92 (28.9) & $38(23.3)$ & $54(34.8)$ & \\
\hline Line of therapy (\% 2 lines) & $236(74.2)$ & $115(70.6)$ & $121(78.1)$ & .126 \\
\hline Baseline SLD, median (range), $\mathrm{mm}$ & $73.5(10-314)$ & $57(10-207)$ & $91(12-314)$ & $<.001$ \\
\hline Number of metastatic sites, median (IQR) & $3(2-4)$ & $3(2-3)$ & $3(2-4)$ & .016 \\
\hline PD-L1 (\% positive) & $169(53.7)$ & $81(50.0)$ & $88(57.5)$ & .181 \\
\hline PD-L1 (\% strong) & $52(16.5)$ & $23(14.3)$ & $29(18.8)$ & .277 \\
\hline EGFR mutation & $28(10.8)$ & $21(12.9)$ & $7(4.5)$ & .008 \\
\hline KRAS mutation & $25(7.9)$ & $16(9.8)$ & $9(5.8)$ & .184 \\
\hline
\end{tabular}

Abbreviations: bTMB, blood-based tumor mutational burden; IQR, interquartile range; SLD, sum of longest diameters. ancluding Asian, black, and others. 


\section{eTable 3. Prognostic Value of bTMB for PFS and OS in Training Set ${ }^{a}$}

PFS

OS

\begin{tabular}{|lcccc}
\cline { 2 - 4 } bTMB & HR $(\mathbf{9 5 \%}$ Cl) & P Value & HR (95\% Cl) & P Value \\
\hline Low $(\leq 7)$ & $0.632(0.498-0.801)$ & $<.001$ & $0.675(0.521-0.874)$ & Ref \\
\hline High $(>7)$ & Ref & & 003 \\
\hline
\end{tabular}

Abbreviations: bTMB, blood-based tumor mutational burden; HR, hazard ratio; OS, overall survival; PFS, progression-free survival.

${ }^{a}$ Adjusted for age, race, sex, smoking status, ECOG performance status, histology, treatment line, baseline sum of longest diameters, number of metastatic sites, PD-L1 expression, and driver mutations.

\section{eTable 4. Characteristics of Patients in Validation Set by bTMB Level}

\begin{tabular}{|c|c|c|c|c|}
\hline Characteristic & $\begin{array}{l}\text { All Patients } \\
\text { n (\%) }\end{array}$ & $\begin{array}{c}\text { Low bTMB (TMB } \leq 7) \\
n(\%)\end{array}$ & $\begin{array}{c}\text { High bTMB (bTMB >7) } \\
\text { n (\%) }\end{array}$ & $P$ Value \\
\hline Total, $n$ & 106 & 49 & 57 & \\
\hline Age, median (range), y & $62.5(36-80)$ & $63(37-77)$ & $61(36-80)$ & .197 \\
\hline Race & & & & .256 \\
\hline White & $89(84.0)$ & $39(79.6)$ & $50(87.7)$ & \\
\hline Nonwhite ${ }^{a}$ & $17(16.0)$ & $10(20.4)$ & $7(12.3)$ & \\
\hline Sex (\% female) & $48(45.3)$ & $27(55.1)$ & $21(36.8)$ & .060 \\
\hline Smoking status & & & & .015 \\
\hline Never & $18(17.0)$ & $13(26.5)$ & $5(8.8)$ & \\
\hline Former/Current & $88(83.0)$ & $36(73.5)$ & $52(91.2)$ & \\
\hline ECOG performance status & & & & .008 \\
\hline 0 & $30(28.3)$ & $20(40.8)$ & $10(17.5)$ & \\
\hline 1 & $76(71.7)$ & $29(59.2)$ & $47(82.5)$ & \\
\hline Histology & & & & .818 \\
\hline Nonsquamous & $68(64.2)$ & $32(65.3)$ & $36(63.2)$ & \\
\hline Squamous & $38(35.8)$ & $17(34.7)$ & $21(36.8)$ & \\
\hline Line of therapy (\% 2 lines) & $76(71.7)$ & $35(71.4)$ & $41(71.9)$ & .954 \\
\hline Baseline SLD, median (range), mm & $78(11-269)$ & $72.5(11-149)$ & $92(38-269)$ & .004 \\
\hline Number of metastatic sites, median (IQR) & $3(2-4)$ & $3(2-4)$ & $3(3-4)$ & .003 \\
\hline EGFR mutation & $5(4.7)$ & $3(6.1)$ & $2(3.5)$ & .527 \\
\hline KRAS mutation & $10(9.4)$ & $6(12.2)$ & $4(7.0)$ & .359 \\
\hline
\end{tabular}

Abbreviations: bTMB, blood-based tumor mutational burden; IQR, interquartile range; SLD, sum of longest diameters.

ancluding Asian, black, and others. 


\section{eTable 5. Prognostic Value of bTMB for PFS and OS in Validation Set ${ }^{\mathrm{a}}$}

PFS

\begin{tabular}{|c|c|c|c|c|}
\hline \multirow[b]{2}{*}{ bTMB } & & \\
\hline & HR (95\% Cl) & $P$ Value & HR $(95 \% \mathrm{Cl})$ & $P$ Value \\
\hline Low $(\leq 7)$ & $0.487(0.315-0.753)$ & .001 & $0.620(0.413-0.931)$ & .021 \\
\hline High (>7) & Ref & & Ref & \\
\hline
\end{tabular}

Abbreviations: bTMB, blood-based tumor mutational burden; HR, hazard ratio; OS, overall survival; PFS, progression-free survival.

${ }^{a}$ Adjusted for age, race, sex, smoking status, ECOG performance status, histology, treatment line, baseline sum of longest diameters, and number of metastatic sites.

\section{eTable 6. Patient Characteristics Before and After PSM}

\begin{tabular}{|c|c|c|c|c|c|c|c|c|}
\hline \multirow[b]{2}{*}{ Characteristic } & \multicolumn{4}{|c|}{ Before PSM } & \multicolumn{4}{|c|}{ After PSM } \\
\hline & $\begin{array}{c}\text { Low bTMB } \\
\text { (TMB } \leq 7) \\
\text { n (\%) }\end{array}$ & $\begin{array}{c}\text { High bTMB } \\
\text { (bTMB >7) } \\
\text { n (\%) }\end{array}$ & SD & $P$ Value & $\begin{array}{c}\text { Low bTMB } \\
\text { (TMB } \leq 7) \\
n(\%)\end{array}$ & $\begin{array}{c}\text { High bTMB } \\
\text { (bTMB >7) } \\
\text { n (\%) }\end{array}$ & SD & $P$ Value \\
\hline Total, $\mathrm{n}$ & 212 & 212 & & & 129 & 129 & & \\
\hline Age, median (range), y & $64(34-85)$ & $63(36-84)$ & 0.10 & .258 & $64(34-84)$ & $64(40-84)$ & $<0.01$ & .886 \\
\hline Race & & & 0.26 & .008 & & & 0.09 & .473 \\
\hline White & 144 (67.9) & $168(79.2)$ & & & $94(72.9)$ & $99(76.7)$ & & \\
\hline Nonwhite ${ }^{a}$ & $68(32.1)$ & $44(67.0)$ & & & $35(27.1)$ & $30(23.3)$ & & \\
\hline Sex (\% female) & $102(48.1)$ & $70(33.0)$ & 0.31 & .002 & $46(35.7)$ & $43(33.3)$ & 0.05 & .694 \\
\hline Smoking status & & & 0.54 & $<.001$ & & & 0.17 & .162 \\
\hline Never & $51(24.1)$ & $12(5.7)$ & & & $13(10.1)$ & $7(5.4)$ & & \\
\hline Former/Current & $161(75.9)$ & $200(94.3)$ & & & $116(89.9)$ & $122(94.6)$ & & \\
\hline ECOG performance status & & & 0.19 & .050 & & & $<0.01$ & 1.000 \\
\hline 0 & $80(37.7)$ & $61(28.8)$ & & & 45 (34.9) & $45(34.9)$ & & \\
\hline 1 & $132(62.3)$ & $151(71.2)$ & & & $84(65.1)$ & $84(65.1)$ & & \\
\hline Histology & & & 0.21 & .035 & & & 0.08 & .510 \\
\hline Nonsquamous & $157(74.1)$ & $137(64.6)$ & & & $88(68.2)$ & $83(64.3)$ & & \\
\hline Squamous & $55(25.9)$ & $75(35.4)$ & & & $41(31.8)$ & $46(35.7)$ & & \\
\hline Line of therapy ( $\% 2$ lines) & $150(70.8)$ & $162(76.4)$ & 0.13 & .186 & $95(73.6)$ & $101(78.3)$ & 0.10 & .382 \\
\hline Baseline SLD, median (range), $\mathrm{mm}$ & $60.8(10-207)$ & $92(12-314)$ & -0.63 & $<.001$ & $74(11-207)$ & $79(12-200)$ & -0.11 & .300 \\
\hline Number of metastatic sites, median (IQR) & $3(2-3)$ & $3(2-4)$ & -0.29 & $<.001$ & $3(2-4)$ & $3(2-4)$ & -0.05 & .333 \\
\hline EGFR mutation & $24(11.3)$ & $9(4.2)$ & 0.27 & .007 & $11(8.5)$ & $6(4.7)$ & 0.16 & .210 \\
\hline KRAS mutation & $22(10.4)$ & $13(6.1)$ & 0.15 & .112 & $9(7.0)$ & $9(7.0)$ & $<0.01$ & 1.000 \\
\hline
\end{tabular}

Abbreviations: bTMB, blood-based tumor mutational burden; IQR, interquartile range; PSM, propensity score matching; SLD, sum of longest diameters. ancluding Asian, black, and others. 
16 - Nie et al

\section{eTable 7. Doubly Robust Estimation for PFS and OS in Propensity Score-Matched Set}

PFS

OS

\begin{tabular}{|c|c|c|c|c|}
\hline Prognostic Factor & HR $(95 \% \mathrm{Cl})$ & $P$ Value & HR $(95 \% \mathrm{Cl})$ & $P$ Value \\
\hline \multicolumn{5}{|l|}{ EGFR (Positive/Negative) } \\
\hline Baseline SLD (>75.5/ $\leq 75.5)$ & & & $1.435(1.056-1.950)$ & .021 \\
\hline \multicolumn{5}{|l|}{ Smoking status (NS/CS+ES) } \\
\hline bTMB (Low/High) & $0.611(0.461-0.810)$ & .001 & $0.593(0.438-0.803)$ & .001 \\
\hline
\end{tabular}

Abbreviations: bTMB, blood-based tumor mutational burden; CS, current smokers; ES, ever-smokers; HR, hazard ratio; OS, overall survival; NS, never smokers; PFS, progression-free survival; SLD, sum of longest diameters.

\section{eTable 8. Treatment Interaction With bTMB in the Combined Set ${ }^{a}$}

PFS

Treatment Low vs High bTMB HR $(95 \% \mathrm{Cl}) \quad$ Interaction $P$ Value

$0.881(0.707-1.097)$

$0.645(0.514-0.808)$

.043

Docetaxel

rar

Abbreviations: bTMB, blood-based tumor mutational burden; HR, hazard ratio; OS, overall survival; PFS, progression-free survival.

aEstimated from a multivariable cox model on the combined dataset. Adjusted for age, race, sex, smoking status, ECOG performance status, histology, treatment line, baseline sum of longest diameters, and number of metastatic sites. 